\title{
RECONFIGURATION ALGORITHMS FOR HIGH PRECISION COMMUNICATIONS IN TIME SENSITIVE NETWORKS: TIME-AWARE SHAPER CONFIGURATION WITH IEEE 802.1QCC
}

\author{
Ahmed Nasrallah ${ }^{1}$, Venkatraman Balasubramanian ${ }^{2}$, Akhilesh S. Thyagaturu ${ }^{3}$, Martin Reisslein ${ }^{4}$, Hesham ElBakoury $^{5}$ \\ 1,2,3,4 Arizona State University, School of Electrical, Computer, and Energy Engineering, 650 East Tyler Mall, Tempe, AZ \\ 85287-5706, USA, ${ }^{3}$ Intel Corporation, 5000 W. Chandler Blvd., Chandler, AZ 85226, USA, ${ }^{5}$ Formerly with Huawei \\ Technologies Co., now Self-employed, 4211 Norwalk Dr., CC-102, San Jose, CA 95129, USA
}

NOTE: Corresponding author: Martin Reisslein, reisslein@asu.edu

\begin{abstract}
As new networking paradigms emerge for different networking applications, e.g., cyber-physical systems, and different services are handled under a converged data link technology, e.g., Ethernet, certain applications with mission critical traffic cannot coexist on the same physical networking infrastructure using traditional Ethernet packet-switched networking protocols. The IEEE 802.1Q Time Sensitive Networking (TSN) Task Group is developing protocol standards to provide deterministic properties, i.e., eliminates non-deterministic delays, on Ethernet based packet-switched networks. In particular, the IEEE 802.1Qcc, centralized management and control, and the IEEE 802.1Qbv, Time-Aware Shaper (TAS), can be used to manage and control Scheduled Traffic (ST) streams with periodic properties along with Best-Effort (BE) traffic on the same network infrastructure. We investigate the effects of using the IEEE 802.1Qcc management protocol to accurately and precisely configure TAS enabled switches (with transmission windows governed by Gate Control Lists (GCLs) with Gate Control Entries (GCES)) ensuring ultra-low bounded latency, zero packet loss, and minimal jitter for ST TSN traffic. We examine both a centralized network/distributed user model (hybrid model) and a fully-distributed (decentralized) 802.1Qcc model on a typical industrial control network with the goal of maximizing the number of ST streams.
\end{abstract}

Keywords - Cyber-physical systems, low-latency traffic, protocol adaptation, reconfiguration, Time Sensitive Networking (TSN).

\section{INTRODUCTION}

\subsection{Motivation}

IEEE 802.1 Time Sensitive Networking (TSN) provides a standardized framework of tools for providing deterministic Ultra-Low Latency (ULL), e.g., for industrial control applications, automotive networking, smart grid applications, and avionics communication systems [11, 22, 30, $34,57,63,88]$. In particular, the IEEE 802.1Qbv Time Aware Shaper (TAS) has received extensive attention as a key tool for achieving a deterministic ULL network service. The TAS operation requires careful planning of the synchronized time cycles $[79,85,91]$ and the gate times that are allocated to the Scheduled Traffic (ST) and the unscheduled Best-Effort traffic (BE). The TAS parameter settings specifying the timing characteristics (cycle time, gate slot allocations) are also commonly referred to as the Qbv schedule or the TAS schedule. For a given static networking scenario, the TAS operation with a properly configured Qbv schedule can ensure the deterministic ULL required by demanding industrial and automotive applications $[8,27,40,59,62,77,84]^{1}$.

Modern network scenarios often involve dynamic changes with varied use cases, such as changes in the network nodes and network topology, or the traffic pattern. For instance, nodes or links may be dynamically

${ }^{1} \mathrm{~A}$ preliminary abridged version of this study appeared in the IEEE Globecom 2019 workshop paper [60]. This journal article substantially extends the prior workshop paper, as explained in Section 1.2. added or removed. Or, nodes may inject additional traffic flows or traffic flows may terminate, or the latency requirements of flows may change dynamically. Such dynamic changes have been included in the use cases defined by the IEC/IEEE 802.1 TSN TG $[10,89]$. In a typical industrial environment, sensors that periodically or sometimes sporadically send ambient measurements to a local gateway require certain Quality of Service (QoS) guarantees $[6,16,31,42,64]$. In such a volatile and dynamic environment, new machinery that requires prioritized execution (e.g., emergency cooling procedures or maintenance tasks for network traffic tests) may be brought onto the factory floor. To deal with such scenarios, the Time-Aware Shaper (TAS) Gate Control Lists (GCLs) in coordination with the Network Management Entities (NMEs), e.g., Centralized Network Configuration (CNC), have to adapt to changing environment conditions by judiciously applying reconfiguration such that stream deadlines and QoS are satisfied.

Generally, in such dynamic networking scenarios, applying only admission control will clearly guarantee (in accordance with a traffic shaper) the QoS metrics of the admitted flows. However, for a given static network configuration, the total number of admissible streams may be well below the number of streams that seek network service. Therefore, adding a dynamic reconfiguration strategy to manage and configure the network appears to be a plausible and attractive solution that intuitively should lower capital and operational expenditures as it mitigates 
the over-provisioning of network resources. The general idea for such an allocation scheme is to control network access in a timely and orderly fashion such that a maximum number of streams can be effectively serviced.

Our objective therefore is to maximize the number of admitted flows (i.e., tasks or streams) in such a dynamically changing and volatile environment whilst keeping the TSN QoS metric guarantees. In this paper, we focus on the IEEE 802.1Qbv [2] enhancements and design a reconfiguration framework taking inspiration from the IEEE 802.1Qcc [3] standards for managing, configuring, and reconfiguring a TSN network.

In IEEE 802.1Qbv, a TAS time slot (corresponding to a GCE and also referred to as slot time) is defined as the portion of the cycle time (CT, which corresponds to the GCL); TAS time slots are allocated to high-priority ST traffic. In our model, the switch/controller computes the TAS time slot for all admitted streams as follows. Essentially, as streams get registered, we keep track of the available remaining capacity, which we set initially to the maximum available capacity on each egress port until the load (which depends on the ST slot size and the cycle time is negative, i.e., oversubscribed link). Such a link oversubscription invokes a procedure call that increases the slot time (by a step size of $1 \%$, or more fine-grained increments) until the remaining load is positive. This procedure is iteratively called until all registered streams and the new stream are appropriately registered with a sufficient ST slot time to transmit all frames during a single appropriately sized CT.

Our proposed TAS configuration/reconfiguration is designed for the centralized (hybrid) model and for the fullydistributed configuration model. In the "hybrid" model, the CNC is utilized for configuration exchanges and network side management, as explained in more detail in Section 3. In the distributed approach, the GCE slot parameters are configured in a distributed manner by the switches as per the distributed algorithm/procedure explained in Section 4. For brevity we refer to the centralized network/distributed user model (hybrid model) also as the centralized model or the centralized topology. We refer to the fully-distributed (decentralized) model also as the decentralized model or the decentralized topology.

\subsection{Related work}

We first note that general performance evaluation strategies for TAS have been explored in $[39,50,73]$ and we follow these strategies in our study. Raagaard et al. $[51,76]$ have presented a heuristic scheduling algorithm that reconfigures TAS switches according to runtime network conditions. Feasible schedules are computed and forwarded using a configuration agent (composed of a Centralized User Configuration (CUC) and Centralized Network Configuration (CNC)). Raagaard et al's model places emphasis on the schedule computation complexity for appearing and disappearing synthetic flows in a fog computing platform. Complementary to this approach, we de- velop comprehensive centralized and distributed reconfiguration frameworks based on firm bandwidth computation strategies that execute at run-time. Further, we conduct a comprehensive performance evaluation of our two frameworks considering common packet flow QoS metrics for both high-priority ST and low-priority BE traffic.

The proposed approach by Nayak et al. [67] exploits the logical centralization paradigm of SDN with real-time traffic to achieve optimal scheduling and routing. Integer Linear Programming (ILP) formulations were used to solve the combined problem of routing and scheduling time triggered traffic. Two main proposals for routing are given, namely $i$ ) scheduling and path-sets routing, and $i i$ ) scheduling and fixed-path routing whereby the ILP formulations are used to find near optimal flow to time-slot allocations. However, the ILP does not scale well with the number of flows, does not provide schedules at runtime speeds, and does not work well with dynamic flow configuration (or reconfiguration). To enhance the architecture proposed by Nayak et al. [67], an augmentation is proposed in [68] that incrementally adds time sensitive flows to the scheduler making the proposed approach reconfiguration capable. Additionally, Nayak et al. $[65,66]$ provide an analysis and evaluation to the problem of flow-span and routing protocol (Equal Cost Multi-Path, and Shorted Path) on transmission scheduling. Further routing refinements have been studied in $[9,48,49,66,72]$.

Focusing on in-vehicular networks, Hackel et al. [38] have proposed a SDN based TSN framework that performs reconfiguration using the Stream Reservation Protocol (SRP) as a means to register and allocate resources for TSN streams. The TSN with SDN is evaluated with two TSN switches and two clients (a sources and sink). In contrast, we provide extensive evaluation for larger network topologies and sources. Using OpenFlow and openPowerLink, Herlich et al. [41] have provided a proof-ofconcept model that highlights the advantages of TSN with SDN and real-time Ethernet protocol. While the model shows promising advantages in theory, only a coarsegrained evaluation was presented that, in contrast to our evaluation, does not examine stream admission rates and TSN QoS. Focusing on remote monitoring and telemetry, Kobzan et al. [46] have presented a solution concept and implementation of an SDN based TSN architecture using IEEE 802.1Qcc. However, the concept is provided without any empirical evaluation. To the best of our knowledge, there are no prior detailed studies on a fluctuating volatile source or a dynamic stream resource allocation and admission control policy in conjunction with a network reconfiguration policy being executed while flows are carried in a TAS time scheduled network. We provide a comprehensive design and evaluation of an SDN based TSN model that bases the specification on the standardization given by the IEEE $802.1 \mathrm{Q}$ standard.

Vlk et al. [87] have proposed a simple hardware enhancement of a switch along with a relaxed scheduling constraint that increases schedulability and throughput of 
the time-triggered traffic but maintains the deterministic nature and timeliness guarantees in a TSN network. Several related scheduling refinements that are orthogonal to the reconfiguration studied in this article have been examined in $[26,40,43,53,74,93]$. We note for completeness that multicast for TSN has been studied in $[80,92]$, while our focus is on unicast traffic.

This article extends the prior conference paper [60], which provided a brief preliminary overview of the decentralized and centralized reconfiguration models, but did not provide the specific operational details, nor detailed performance evaluations. This present journal article provides the full operational details as well as comprehensive performance evaluations.

\subsection{Contributions}

We comprehensively evaluate the performance of TAS for reconfigurations in the hybrid and fully distributed models with respect to network deployment parameters, such as the time period for the Gate Control List (GCL) to repeat (whereby the duration of one GCL repetition corresponds to the $\mathrm{CT}$ ), the gating ratio proportion, i.e., Gate Control Entry (GCE) proportion, to control the delay perceived at the receiving end, the signaling impact on ST and $\mathrm{BE}$ classes, and the packet loss rate experienced at the receiving end. In particular, we make the following contributions:

i) We design a CNC interface for a TSN network to globally manage and configure TSN streams, including admission control and resource reservation.

ii) We integrate the CNC in the control plane with TAS in the data plane to centrally manage and shape traffic using the CNC as the central processing entity for flow schedules as more flows are added.

iii) We modify and test the model to operate in a distributed fashion, i.e., the signaling is conducted inband and the control plane processing is conducted at the individual distributed switches.

iv) We evaluate each design approach for a range of numbers of streams with different TAS parameters. We show results for admission ratios, network signaling overhead, and QoS metrics.

\subsection{Organization}

This article is organized as follows. Section 2 provides background information and an overview of related work on the 802.1 TSN standardization, focusing on the enhancements to ST as well as centralized management and configuration. Section 3 shows the complete top-down design of the CNC (hybrid model) and the main components that achieve ultra-low latencies and guaranteed QoS for a multitude of ST streams. Similarly, Section 4 shows the approach used in implementing the decentralized (fully distributed) TAS reconfiguration model. The simulation setup as well as main parameters and assumptions are given in Section 5 and results are presented in Section 5.2 and Section 5.3. Finally conclusions and future work are outlined in Section 6.

\section{BACKGROUND: IEEE 802.1 TIME SENSI- TIVE NETWORKING}

\subsection{IEEE 802.1Qbv: Time Aware Shaper (TAS)}

TAS's main operation is to schedule critical traffic streams in reserved time-triggered windows. In order to prevent lower priority traffic, e.g., BE traffic, from interfering with the ST transmissions, ST windows are preceded by a so-called guard band. TAS is applicable for Ultra-Low Latency (ULL) requirements but needs to have all timetriggered windows synchronized, i.e., all bridges from sender to receiver must be synchronized in time $[79,85]$. TAS utilizes a gate driver mechanism that opens/closes according to a known and agreed upon time schedule for each port in a bridge. In particular, the Gate Control List (GCL) represents Gate Control Entries (GCEs), i.e., a sequence of on and off time periods that represent whether a queue is eligible to transmit or not.

The frames of a given egress queue are eligible for transmission according to the GCL, which is synchronized in time through the 802.1AS time reference. Frames are transmitted according to the GCL/GCE and transmission selection decisions. Each individual software queue has its own transmission selection algorithm, e.g., strict priority queuing. Whereby, a software queue is the queue before the NIC hardware queue takes ownership of the currently forwarded frame in an 802.1 switch. Overall, the IEEE 802.1Qbv transmission selection transmits a frame from a given queue with an open gate if: $(i)$ The queue contains a frame ready for transmission, $(i i)$ higher priority traffic class queues with an open gate do not have a frame to transmit, and ( $i i i)$ the frame transmission can be completed before the gate closes for the given queue. Note that these transmission selection conditions ensure that low-priority traffic is allowed to start transmission only if the transmission will be completed by the start of the ST window for high-priority traffic. Thus, this transmission selection effectively enforces a "guard band" to prevent low-priority traffic from interfering with highpriority traffic [30].

\subsection{IEEE 802.1Qcc: centralized management and configuration}

IEEE 802.1Qcc [3] provides a set of tools to globally manage and control the network. In particular, IEEE 802.1Qcc enhances the existing Stream Reservation Protocol (SRP) with a User Network Interface (UNI) which is supplemented by a Centralized Network Configuration (CNC) node. The UNI provides a common method of requesting layer 2 services. Furthermore, the CNC interacts with the switch UNI to provide a centralized means for perform- 
ing resource reservation, scheduling, and other types of configuration via a remote management protocol, such as NETCONF [25] or RESTCONF [12]; hence, 802.1Qcc is compatible with the IETF YANG/NETCONF data modeling language.

The IEEE 802.1Qcc standard specifies three models for configuring the Time-Aware Shaper (TAS) gating schedules (GCL/GCE timing): a fully-centralized model, a centralized network/distributed user model (hybrid model), and a fully-distributed configuration model. The centralized model greatly eases control and configuration messages sent across the network and can precisely configure TAS schedules due to having the complete knowledge of the network and the full capabilities of each bridge. However the centralized model suffers from common disadvantages, such as a single-point of failure, relatively large capital/operational (CapEx/OpEx) expenditures (as the centralized control may be superfluous in a small-scale network [15]), and adding unnecessary complexity to a small-scale network.

Compared to the centralized network/distributed user model (hybrid model), the fully centralized model does not add any benefits for the reconfiguration approach towards enhancing the resource allocation and QoS nor does it allow better deterministic forwarding. The main usage for the CUC is to take into account the application's complex timing and computation requirements for industrial applications which is out of scope for our evaluation. Rather, our focus is on the reconfiguration for proper resource allocation. Therefore, we focus on the centralized network/distributed user model (hybrid model) form of the centralized model in this study.

A fully-distributed configuration model (e.g., SRP over MRP or RAP over LRP) may be attractive for some networks. The fully-distributed configuration model avoids the added complexity and single point of failure of a centralized management entity. Moreover, Chen et al. [15] have argued that the centralized configuration models can be an over-design for real-time applications with relaxed latency requirements (order of magnitude of milliseconds). Chen et al. have also argued that the distributed model is more scalable. (However, studies of the fully distributed model with RAP over LRP targeted typically applications with relatively relaxed latency requirements.)

In the absence of a Centralized Network Configuration (CNC) node, the TSN Task Group (TG) specifies the IEEE 802.1CS (Link-Local Registration Protocol, LRP) [29] standard for registration and distribution of application configuration parameters over point-to-point links targeting newly published TSN features. A legacy protocol, such as the Stream Reservation Protocol (SRP) [1] which is primarily used for Audio-Video Bridging (AVB) applications, is intended to serve as the main resource reservation and admission control protocol. However, extending and porting the SRP to be utilized for bridges that support TAS will not suffice since bandwidth reservation cannot directly apply TAS's time slot reservation natively. There- fore, the Resource Allocation Protocol, IEEE 802.1Qdd (RAP) [15], has been proposed to apply a distributed resource reservation that can exchange TSN features.

\section{HYBRID MODEL DESIGN AND FRAME- WORK CONSIDERATIONS}

This section presents our design methodology and main signaling framework for the centralized network/distributed user model (hybrid model). Our main goals behind designing the CNC are given by the following constraints. Additionally, the CNC can be logically or physically connected to the data plane with in-band or out-of-band management links. With in-band communication under the hybrid model, only one switch is physically connected to the $\mathrm{CNC}$; thus, signaling packets between the switches and CNC affect data traffic similar to the distributed approach, but the CNC still functions as the centralized configuration. For the hybrid model evaluations in this study, we consider out-of-band communication, i.e., all switches are physically attached to the CNC.

1. Our focus is mainly on stream based network adaptation. By this technique, fluctuating streams (already registered streams and new incoming streams) and their requirements can be accommodated by the network dynamically based on a single remote procedure call to the CNC.

2. We identify and execute flow requirements by populating the registration table. The control plane resource orchestration is purely carried out by monitoring existing flows which have been satisfied.

3. We conduct resource allocation based on the stream network resource utilization.

Our main assumption to accurately apply admission control and, consequently, reconfiguration, is that each source must define a flow in terms of total resources needed (governed by the bandwidth requirements) and the total time needed for the resource to be used (which in our traffic model is termed the resource utilization time). Essentially, the CNC uses this information (which is tagged in the Ethernet frame header) to determine whether a stream (flow) is admitted or rejected.

\subsection{Core components}

Our design is split into two layers, Control Plane and Data Plane, following the decoupling SDN paradigm, thereby inheriting the benefits of the orthogonality of the two planes, as shown in Fig. 1.

\subsubsection{Configuration module}

The configuration module is the main component that interacts with the registered flows and network components. It includes the global stream registration table which records all approved streams transmitting in 


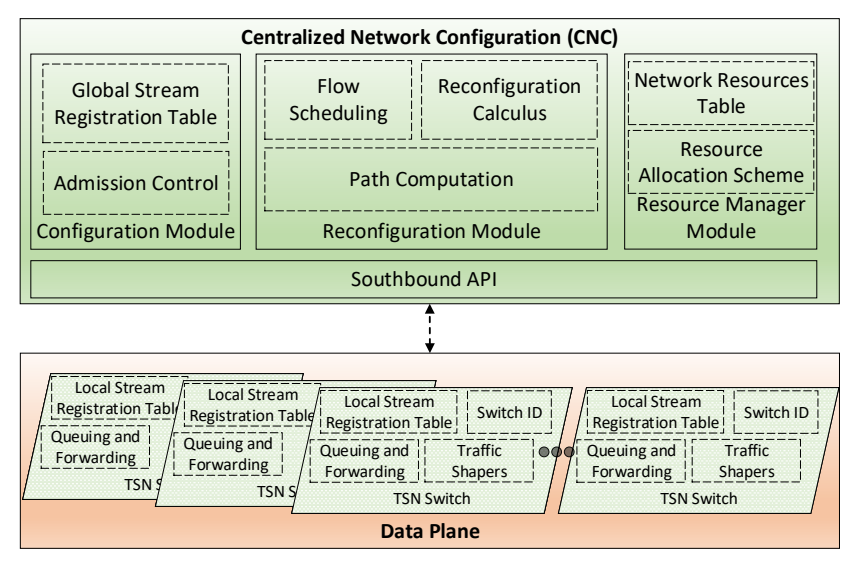

Fig. 1 - Network Management Entity Framework for TSN Switches: Centralized Network Configuration (CNC) is used to send and receive Control Data Traffic (CDT), which we define as the signaling traffic, e.g., the UNI information to and from the CNC and switches/sources or LLDP discovery packets, to configure routing segments and network resources.

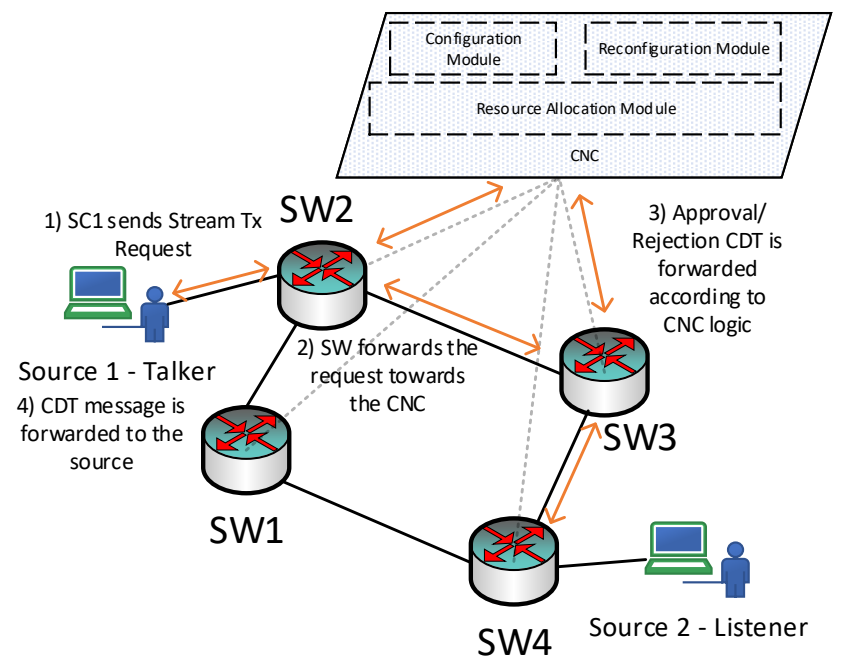

Fig. 2 - Centralized Model Example: Source 1 sends a CDT stream request to its gateway. The gateway forwards it to its governing CNC. The CNC decides if the stream will be serviced according to the source UNI. All switches in the explicit path for the stream are notified if the stream is accepted. Otherwise, the gateway is alerted of the rejection. Lastly, the gateway forwards it back to the source which prompts the source to start sending data ST traffic in the next available cycle (if approved).

the network (i.e., currently utilizing network resources (bandwidth)), and the admission control element that encapsulates and decapsulates CDT headers and forwards the information to the necessary module/element.

Global stream registration table The source streams (devices/users) make a Remote Procedural Call (RPC) via the stream registration interface for providing information that can be mapped as a unique tuple structure identification $<$ FlowID, BridgeGateway $>$. Upon receiving the registration packet, i.e., Control Data Traffic (CDT), the CNC determines whether the new stream can be accepted in its stream table. To guarantee the QoS for all registered streams, admission control principles are applied to all streams according to the stream's path, required network resources, and available resources. Fig. 2 shows an exam- ple where the source sends a CDT stream request to the gateway switch, which is then forwarded to the CNC for admission control and resource reservation.

Admission Control The admission control element is the first element that the new streams interacts with. The admission control element in the configuration module globally manages all streams transmitting in the TSN domain governed by the CNC. The admission control element extracts the necessary information from the CDT packet and forwards the information according to the CDT type. The CNC applies several steps to decide whether to accept or reject the stream transmission request.

1. The CNC checks the destination address(es) of the stream and consults its resource manager module for network resources available on the new stream's path, which is computed based on the path computation element within the CNC.

2. According to the bandwidth required for the new stream (calculated at the bridge gateway for the new stream as the stream packet rate multiplied by the packet size and divided by the ST slot time), all links on the path are checked to see if enough bandwidth is available for the new stream.

3. In the event that not enough resources are available, the CNC applies the TAS reconfiguration module to identify the bottleneck link(s) and to check whether the gating ratio can be increased for that specific traffic class whose current resource utilization will not exhaust the resources by being added to the TAS slot reservation.

\subsubsection{Reconfiguration module}

The reconfiguration module includes the flow scheduling element (for our network model, the Time-Aware Shaper (TAS) is used in the data plane), the reconfiguration calculus element which optimizes flow registration according to each stream's total resource utilization and flow deadlines, and finally the path computation element which defines the path for all streams according to the QoS constraint.

Flow scheduling The flow scheduling element currently takes the Time-Aware Shaper into consideration. Due to the TAS's requirements on time synchronization between network components (switches, hosts, etc.), the CNC follows the same principle of scheduling flows according to a known timescale (initially set to be $50 \mu \mathrm{s}$ in our network model). The CNC then passes on this time synchronization information to the TSN enabled switches within its domain. Any approved streams will transmit frames according to the time scale specified by the flow scheduler in the CNC. 
Reconfiguration calculus In addition to centrally managing resources and providing admission control policies to the network, the CNC can invoke the TAS reconfiguration strategy with the goal of borrowing BE time slots for pending ST traffic streams. This element consults the resource manager module on the bottleneck link and checks whether the added stream will oversubscribe the link. The TAS reconfiguration incrementally ( $1 \%$ of total CT) increases the traffic class slot time and reserves it for the new stream.

Path computation For large scale and complex LAN/MAN topologies, it is often required to supplement streams with equal cost paths in the event of a path disruption (e.g., link failure, stream saturation, and explicit congestion). The CNC's path computation element is tasked with finding such paths as a fail-over approach to avoid any violations to any stream's QoS. Presently, our model has a rudimentary application of path computation, i.e., it is defined statically for all core network components (shortest path), since our main emphasis in this study is on reconfiguration based on stream characteristics as defined by the source.

\subsubsection{Resource manager module}

The resource manager module centrally manages all network resources within the CNC's domain. It includes the network resource table that records all streams' usage of resources, and the resource allocation scheme element to which we delegate the task of calculating the required network resources for a given stream according to an allocation scheme.

Network resource table To remove certain overheads on the configuration module, the network resource table operates in tandem with the global stream registration table to accurately determine the required network resources (mainly bandwidth for our traffic model). It classifies streams based on periodic stream properties. Any stream that has been approved by the CNC has a record attached to it in the network resource table.

Resource allocation scheme Several allocation schemes can be implemented for all traffic classes defined in the network. For periodic streams, the time slot given by the flow scheduler (according to the TAS Cycle Time and number of traffic classes) and the data rate defined by the source is used to calculate the required bandwidth for each link on the path to the destination (i.e., sink).

\subsubsection{Data plane}

The data plane contains all core switches. Any TSN switch interfaced by the CNC is given a switch ID and has a local stream registration table. The remaining switch elements

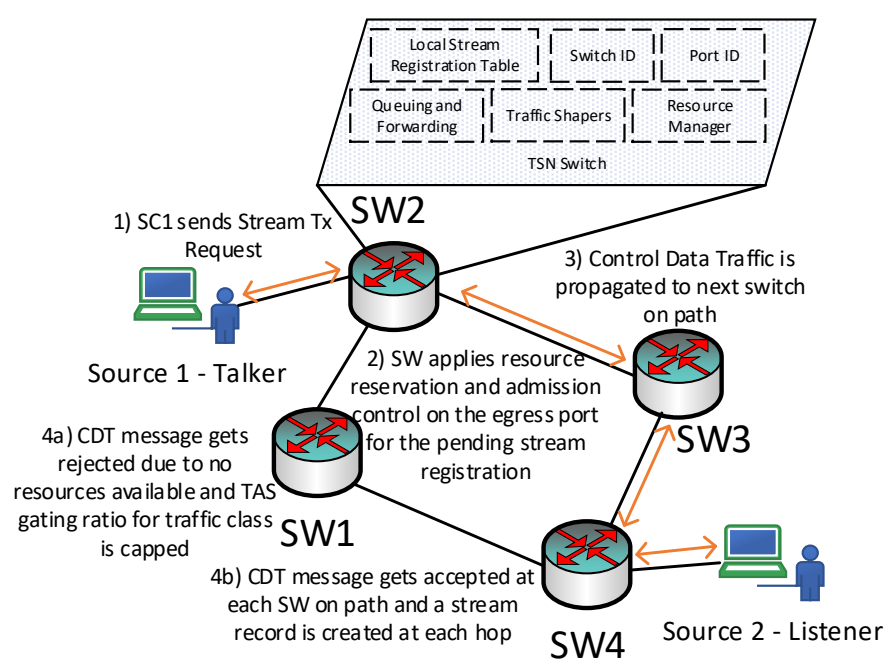

Fig. 3 - A TSN fully distributed configuration model example illustrating the general strategy and logic of each TSN switch with TAS support. In the absence of a CNC to centrally manage network parameters, each switch performs admission control and resource reservation (according to the TAS time slot load) and propagates the information to the next hop on the stream path. A single rejection on one hop terminates the forwarding of the CDT, and sends another CDT on the reverse path indicating the stream rejection outcome. If all switches on the path accept the stream, then the source is notified of the stream acceptance outcome and can begin forwarding in the next TAS cycle. In our model, CDT traffic has higher priority than non-CDT traffic (including ST). The formal definition of the CDT traffic is left for future work.

compose the forwarding and queuing operation with several traffic shapers (802.1Qbv TAS in our network model).

Local stream registration table This data plane registry contains the subset of source streams that are established for the corresponding bridge gateway and attached sources to each port. The CNC delegates some control to the bridge gateway to instruct and alert sources of any new network conditions and explicit changes.

Traffic shaper - Time-Aware Shaper (TAS) The TAS is the main shaping and scheduling mechanism that controls the gating schedules for all the traffic classes within the TSN domain (which is considered to be equivalent to the CNC domain). All bridges are synchronized to the same gating schedule GCL Cycle Time (CT) given by the CNC's flow schedule element (CT indicates the time period for the GCL to repeat).

\section{DECENTRALIZED MODEL DESIGN AND FRAMEWORK CONSIDERATIONS}

This section presents our design methodology and framework for the TAS reconfiguration in the decentralized (fully distributed) model. Our current proposed architecture generally follows the steps enumerated below and illustrated in Fig. 3. Our description focuses on the additions to the design of RAP over LRP, e.g., TAS slot computation/reservations. 
1. At each egress port (Port Identifier, PID), the TSN switch maintains a local stream registration table that includes information, such as flow ID, gateway (i.e., the first bridge that a talker is connected to), destination address(es), the traffic injection rate per GCL cycle time, and the calculated port bandwidth requirement. The traffic injection rate is not computed, rather the traffic injection rate is reported by the source (talker) to the network devices. It mainly indicates the bandwidth requirements of a stream. Bandwidth for a bridge egress port needed for a stream is computed using the ST injection rate (or ST rate), the average packet size, and the bridge TAS timing configuration (e.g., the CT and current traffic class slot time). This information is carried and communicated between bridges using the CDT packet type identifier (or message type).

2. A source (talker) can send a stream transmission request, i.e., a CDT message of type Stream Transmission Request, to register its stream and to use the TSN service for ST.

3. Each switch maintains a resource manager module for each port. If the newly incoming stream is accepted (due to available resources and TAS slot space). The TAS slot size for a specific traffic class is governed by the CT and traffic class gating ratio (in time). The TAS ST slot can be configured/reconfigured according to stream requests and terminations. The stream registration message is then propagated towards the next switch, and a map is maintained for the stream (and any other streams) pending approval.

4. If accepted by the last switch, then the stream registration record is added to the local stream registration table, and bandwidth resources are allocated for the stream and TAS slot space is modified (if necessary) on the reverse path. The main reason for allocating the resources in the reverse path is as follows. If we allocate the resources in the forward direction but a switch in the next hop rejects the stream (due to lack of resources), then we have to release the resources reserved earlier for the stream. Therefore, we avoid the allocation until all hops provide assurance that the stream will be accommodated.

5. Each switch receiving the pending registration message adds the stream record to its local table, allocates the necessary resources and TAS slot reservation, and propagates the registration message towards the source gateway.

6. The source gateway receives the pending stream registration message and similarly allocates the resources and finally sends an approval granted message towards the source, which prompts the source to start sending data in the next available TAS cycle.

\subsection{Core components}

This section outlines the main components required to successfully implement stream admission control and resource reservation within switches that support the TAS traffic shaper in a distributed fashion. Fig. 4 illustrates the typical registration/reservation procedure for all streams within the TSN domain.

\subsubsection{Admission control}

The admission control element extracts the necessary information from the CDT packet and forwards the information according to the CDT type. The switch forwarding mechanism applies several steps to accurately decide whether to accept or reject the stream transmission request. Note that the stream transmission request corresponds to a CDT request. The switch consults the resource manager module to check if enough resources (bandwidth) is available for the new stream. In particular, a given stream's bandwidth requirement is calculated by multiplying the ST injection rate with the average packet size and dividing by the current ST slot size. Note that the traffic class TAS slot time is the time during which the TAS gate is open to transmit frames belonging to the considered class. Also note that all GCEs are executed during each CT. If the CT is smaller than the aggregate of the GCEs, then we need to either increase the CT or reject streams that cause the exceedance of the CT.

\subsubsection{Flow scheduling}

This element currently takes the Time-Aware Shaper into consideration. Due to the TAS's requirements on time synchronization between network components (switches, hosts, etc.), all switches/hosts follow the TAS GCL timescale cycle time (e.g., $50 \mu \mathrm{s}$ ). Depending on the number of supported traffic classes, the TAS cycle time can be divided into appropriate slots for each traffic class load. The TAS CT is divided among all the traffic classes (in our evaluation model, we consider two traffic classes, BE and ST). Currently, in our evaluations, the CT is initially predefined to 50 microseconds. Note that the CT could be changed/configured dynamically. The dynamic adaptation of the CT with respect to new stream additions, application specifications, or other events is a topic for future research.

\subsubsection{Stream registration table}

In our evaluations, stream creation follows a Poisson process with a prescribed stream generation rate $\pi$. Different scenarios with varying mean stream lifetimes (durations) enable analysis of how reconfiguration works in multiple settings. The stream registration table contains the characteristics of the source streams that are established for the corresponding bridge egress port. Each record gets populated (if accepted) on the reverse path taken by the stream's registration message (after reaching the destina- 


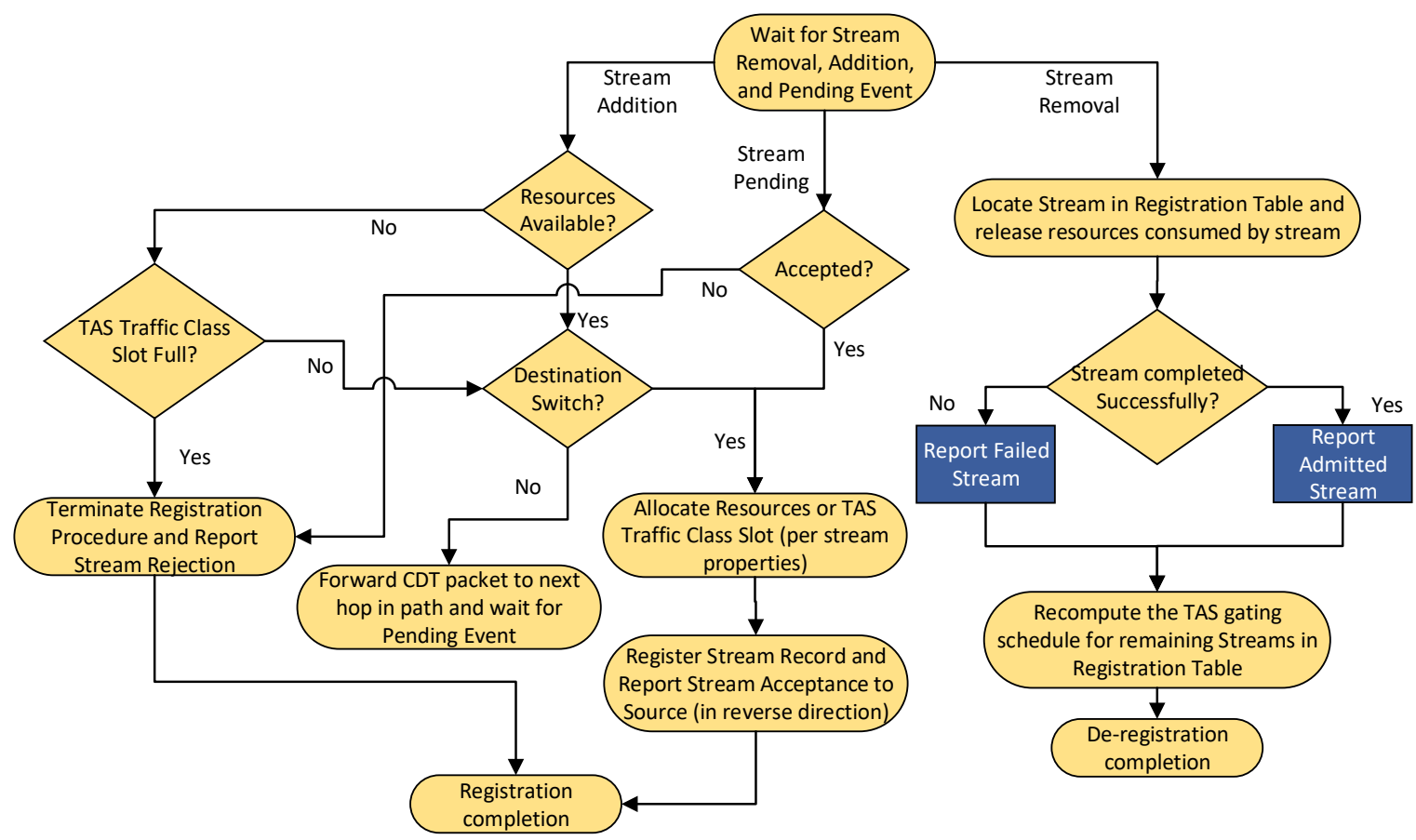

Fig. 4 - The main logical steps performed by each switch along the stream's path are shown to apply stream registration and reservation. Each switch generally waits for an event (addition, removal, or pending) for each stream. For instance, a stream removal is usually based on the resource utilization time (stream lifetime) that was specified at stream establishment. The bridges that allocated resources for the stream can remove the stream after the stream lifetime has expired. For the cases of stream addition or pending, the event is the CDT message received (whether in the forward or reverse direction). Towards completing (finalizing, confirming) a stream reservation (registration), the pending event is the event when a CDT message is received in the reverse direction where each switch (not the last switch) waits for the approval (confirmation of reservation) of the next-hop switch.

tion switch).

\subsubsection{Traffic shaper - Time-Aware Shaper (TAS)}

The TAS is the main shaping and scheduling mechanism that controls the gating schedules for all the traffic classes within the TSN domain. All bridges are synchronized to the same gating schedule GCL CT that is initially predefined by the network administrator. Ideally, we want the CT to be large enough for all streams from all traffic classes to be accommodated and short enough that all streams meet their delay requirements. In our current evaluations, the CT is predefined at 50 microseconds.

\subsubsection{Reconfiguration calculus}

The reconfiguration (dynamic configuration) of the TAS schedules (switch GCL/GCE) for each egress port is dynamically invoked according to two principle events, $i$ ) adding a new stream, and $i i$ ) removing an existing stream. The switch's gating ratio (for a particular stream belonging to a defined traffic class) reports certain parameters (e.g., packet injection rate, maximum packet size, latency requirement, deadline, and application response time) which are then used to check if enough slot time is available (which corresponds to attempting bandwidth reservation). In the event that no slots are available, the GCE slot size is recomputed (according to the registered stream properties within the registration table), generally by allocating more resources from BE Traffic. The stream lifetime is reported by the source to the network as User/Network Information (UNI). Each UNI is propagated by each switch along the path which allows the switch to register the stream and store the stream's resource utilization time (stream lifetime) among other critical information. Any information pertaining to the UNI of a stream is recorded in the stream registration table. In terms of GCEs for TAS with the support of ST and BE traffic classes, only two GCEs within a GCL (1/0 (ST/BE) for the first entry and 0/1 (BE/ST) for the second entry) are necessary with a total of three outbound queues for each egress channel port in a TSN switch; two queues for each traffic class (ST and BE), and another queue for CDT traffic (signaling traffic).

Upon initialization, each switch allocates $20 \%$ of the CT to ST traffic, and BE traffic is initially allocated the remaining $80 \%$ of the CT. These initial settings are chosen arbitrarily to start up the network system. As streams get registered, the ST slot time is recomputed (according to the stream packet size, ST injection rate, and current slot time). If the stream is the first stream to the switch, then the ST slot size is set at a minimum to $11 \%$ (a minimum of $1 \%$ step size for the added ST flow plus the minimum ST partition of $10 \%$ ) of the CT. Thus, as ST streams are admitted and exit the system, the ST vs. BE allocation is dynamically adapted in the reconfiguration scenarios. The minimum step size of $1 \%$ of the CT is considered so as to limit the adaption granularity to a reasonable level. Note that the ST to BE slot size (or gating ratio) is limited to 


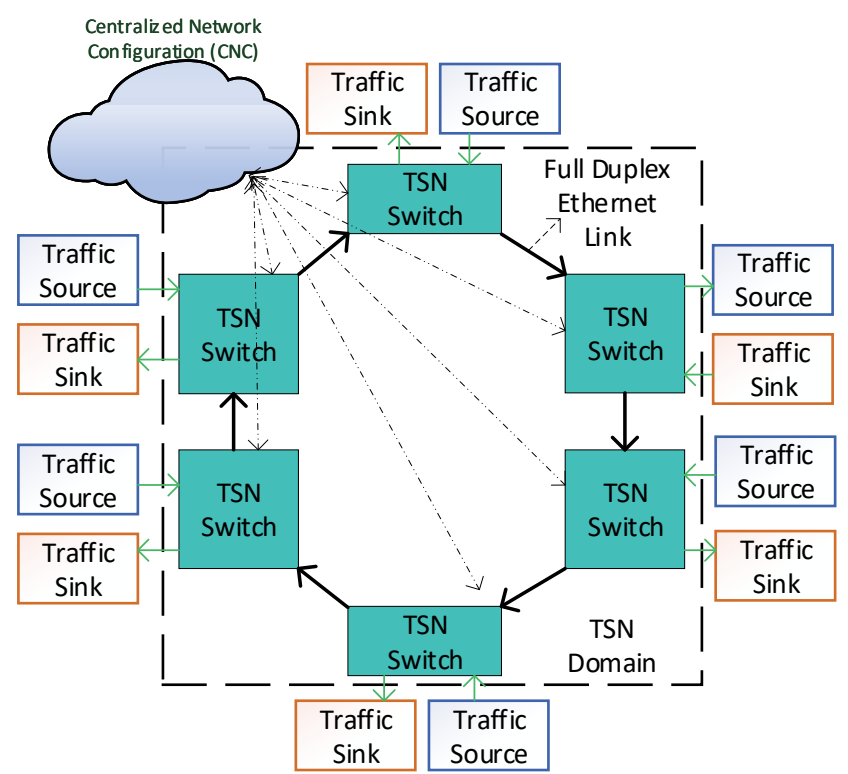

Fig. 5 - Industrial control loop topology [36]: Each source generates stream data with varying hop counts and packet rates unidirectionally or bidirectionally across the six switches ultimately destined to a sink

$10 \%$ and $90 \%$ for the lower and upper limits, respectively. The main reasoning behind this design choice is to avoid any starvation of lower priority traffic.

\subsubsection{Path computation}

While a path computation module is fundamentally necessary in any switch (in a decentralized/distributed network), we define static shortest path routing tables for destination addresses and associated ports on each switch. Essentially, we assume a procedure to compute paths, i.e., we assume that there is a path computation module, e.g., Path Computation Engine (PCE), that is used in both centralized and distributed configuration models (the path computation can be accelerated with hardware modules $[47,70,83]$, if needed). We make this assumption to simplify operations and place emphasis on the TAS reconfiguration technique.

\subsubsection{Network resource table}

To remove certain overheads of the configuration procedure, the network resource table operates in tandem with the stream registration table to accurately determine the required network resources (mainly bandwidth for our traffic model) per switch egress port. The network resource table classifies streams based on periodic and sporadic stream properties, though currently our focus is on periodic ST streams. Any stream that has been approved by a switch has an associated record in the network resource table, located within each switch, which can be called to compute and store current and remaining link/port loads for each switch. Each egress port has a network resource table.
Table 1 - Simulation parameters

\begin{tabular}{|c|c|c|}
\hline Key & Symbol & Value \\
\hline $\begin{array}{l}\text { Simulation Dura- } \\
\text { tion }\end{array}$ & Sim $_{\text {limit }}$ & 100 seconds \\
\hline $\begin{array}{ll}\text { Initialized } & \text { Cycle } \\
\text { Time } & \end{array}$ & $G C L_{C T}$ & $50 \mu \mathrm{s}$ \\
\hline $\begin{array}{l}\text { Initialized Gating } \\
\text { Ratio }\end{array}$ & $S T_{\text {init }}^{R}$ & $20 \%$ (i.e., $10 \mu$ s) \\
\hline $\begin{array}{l}\text { Average Streams } \\
\text { per Second }\end{array}$ & $\pi$ & $1-20$ \\
\hline $\begin{array}{l}\text { Average stream du- } \\
\text { ration }\end{array}$ & $\tau$ & $2-5$ seconds \\
\hline BE Traffic Intensity & $\rho_{L}$ & $\begin{array}{l}0.1,1.0,2.0 \text { Gbps } \\
\text { (580 byte packets) }\end{array}$ \\
\hline ST sources & $S$ & 6 \\
\hline Queue Size & $Q_{\text {size }}$ & $512 \mathrm{~KB}$ \\
\hline
\end{tabular}

\section{PERFORMANCE EVALUATION}

\subsection{System overview and simulation setup}

This section explains the simulation setup and model. Furthermore, the topology and simulation scenarios will be presented. Throughout, we employ the OMNet++ [86] simulation environment. For each evaluation for a given set of parameters, we conduct 5 independent simulation replications; each replication simulates the network for 20 seconds. The widths of the resulting 95\% confidence intervals are smaller than $5 \%$ of the corresponding sample means and are therefore omitted from the plots to avoid clutter.

\subsubsection{Network model}

The network topology is modeled around an industrial control loop topology that consists of six core switches in a ring topology. In the case of the centralized model, a CNC is used with out-of-band connections to each of the core switches; while in the distributed approach, the signaling is in-band and can interfere with data traffic within the TSN domain, as shown in Fig. 5. Each switch-to-switch link operates as a full-duplex Ethernet link with a capacity (transmission bitrate) $R=1 \mathrm{Gbps}$. Each switch can act as a gateway for a number of traffic sources and one sink. The distance between two successive switches along the ring is fixed to $100 \mathrm{~m}$ and the switch-to-switch propagation delay is set accordingly to $0.5 \mu \mathrm{s}$. The out-of-band connections have exactly the same configurations as the normal full-duplex Ethernet links in the data plane, i.e., the same bitrate and propagation delay. All switches are configured to use $802.1 \mathrm{Qbv}$ TAS as the traffic shaper for each switch-to-switch egress port whose flow schedule (ST gating ratio and cycle time) is configured by the CNC in the centralized (hybrid) model and independently in the decentralized (fully distributed) model. For all simulation runs, the ST slot size is initialized to $20 \%$ of the CT. For the operation without reconfiguration, the ST slot size is kept at $20 \%$ of the CT; whereas, for the operation with reconfiguration, the ST slot size is dynamically recomputed when the first stream transmission request arrives. 


\subsubsection{Traffic model}

We consider periodic (pre-planned) traffic for the ST traffic and sporadic (random) Poisson traffic for the BE traffic. To emulate dynamic conditions in the network, we employ several distributed ST sources that generate $\pi$ ST streams according to the network and traffic parameters shown in Table 1. The stream generation follows a Poisson process with a prescribed mean rate of $\pi$ generated streams per second. We refer to the stream generation rate $\pi$ also as the stream mean rate $\pi$. Each ST stream injects one packet of size 64 bytes per cycle. A destination address is assigned by the number of switch-to-switch hops. A given stream that has been generated at the traffic source attached to a given TSN switch is destined to the traffic sinks at the other five TSN switches with a uniform probability of $1 / 5$. Furthermore, at stream creation, each stream is given a start time (usually the current runtime), and a finish time according to a stream lifetime (duration) that follows the exponential distribution with mean $\tau$. The exponential stream lifetime is considered as call level dynamics in communication networks often follow Poisson process dynamics, i.e., exponential call lifetimes. As TSN networks become more commonly deployed, it will be important to verify the stream lifetime dynamics through real system measurements.

We consider admission as the completion of the reservation of the network resources for the flow from the source node to the destination node. Each source is attached to a core TSN switch gateway (first hop switch). While the TSN switches operate with time synchronization, the ST sources (outside the TSN domain) do not need to be synchronized. However, note that the ST traffic follows an isochronous traffic class, as specified by IEC/IEEE 60802 , whereby the sources are synchronized with the network after stream registration is completed. In particular, the ST sources inject the ST traffic in just-in-time fashion, i.e., the transmission of the ST packets out of a source starts at the instant of the start of the ST transmission slot at the switch that is directly attached to the source. Packets are time stamped for the packet delay measurement at the time instant when the packet transmission out of the source commences.

\subsection{Centralized (hybrid) model evaluation}

In evaluating the proposed solution described in Section 3, we consider both periodic ST traffic and sporadic BE traffic, as described in Section 5.1.2. We evaluated the CNC with TAS shaper on the industrial control loop for the unidirectional and bidirectional topologies and results are collected for the simulation parameters shown in Table 1.

\subsubsection{Unidirectional ring topology}

Fig. 6 shows the average mean delay for ST traffic and for $\mathrm{BE}$ traffic for the centralized unidirectional ring topologies. The average delays are generally short and stable

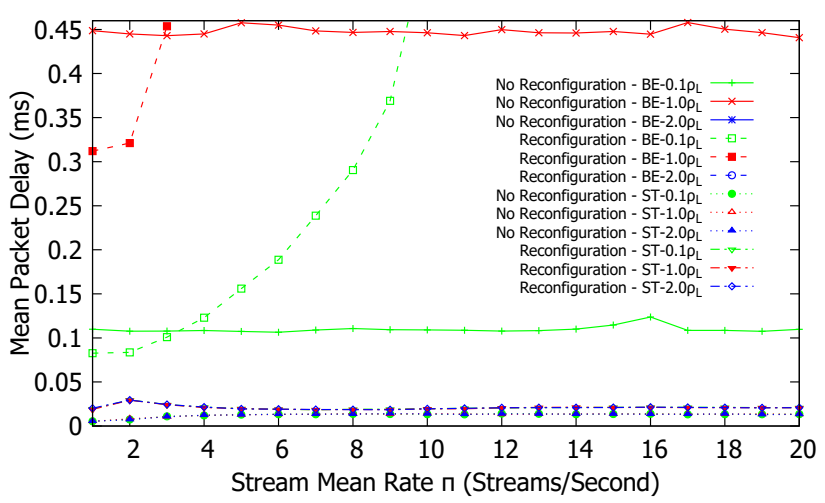

(a) $\tau=2$

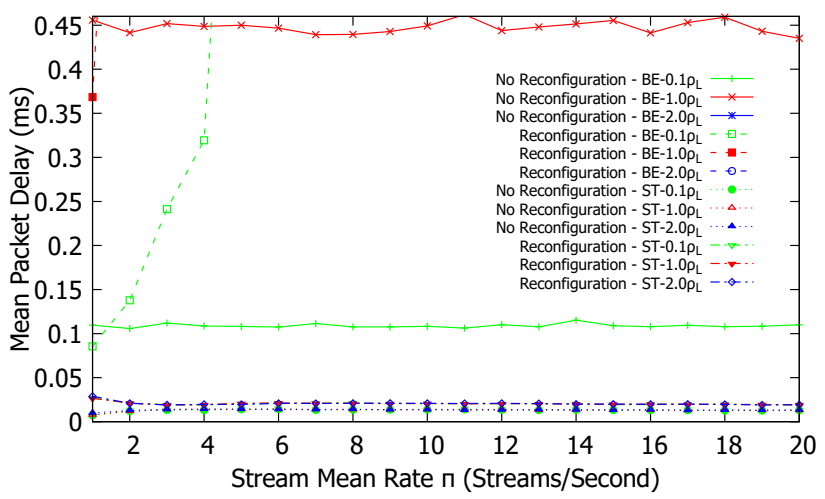

(b) $\tau=5$

Fig. 6 - Centralized (Hybrid) Unidirectional Topology: Mean end-to-end delay for ST and BE traffic for mean stream durations $\tau=2$ and $\tau=$ 5 seconds under different BE loads $\rho_{L}$, and ST stream rates $\pi$.

for both BE and ST traffic. Since the CNC manages the ST traffic streams and therefore guarantees the bandwidth rates needed across the stream's path, the ST delays are less than $100 \mu$ s for all average stream durations $\tau$. The ST streams with reconfiguration at the CNC experience higher delays than for the no reconfiguration scenarios since we essentially push more ST traffic into the network which increases the queuing delay. BE traffic experiences much higher delays than ST. With the no reconfiguration approach, the BE traffic delay is nearly constant since the gating ratio is left unchanged. The $\mathrm{BE}$ mean delay increases dramatically (up to $21 \mathrm{~ms}$ ) with reconfiguration since the accepted ST streams tend to consume the full permitted $90 \%$ of the CT, leaving only very limited transmission resources for $\mathrm{BE}$ traffic.

As mentioned in the introduction section, TSN needs to limit the maximum delay in order to deterministically forward traffic across a TSN domain. Fig. 7 shows the maximum delay for the ST traffic. We observe from Fig. 7 that for the unidirectional ring topology with a maximum of five hop streams, the maximum delay for the reconfiguration approach is below $0.11 \mathrm{~ms}$. On the other hand, for the "no reconfiguration" approach, the maximum delay is below $60 \mu$ s due to lower frame residence time on each switch; however, reconfiguration increases the admission of ST streams as examined next in Fig 8. TAS in conjunction with the $\mathrm{CNC}$ registration and reservation 


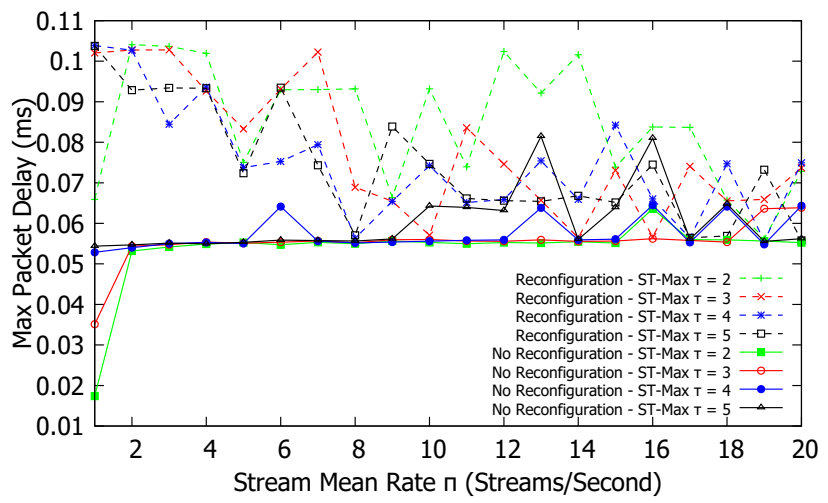

Fig. 7 - Centralized Unidirectional Topology: Maximum packet delay for TAS with centralized configuration (CNC) management.

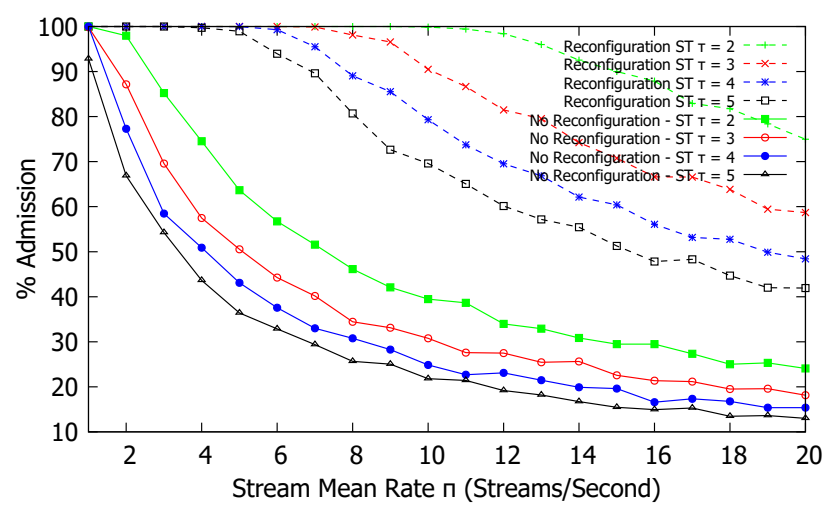

Fig. 8 - Centralized Unidirectional Topology: Stream Admission percentage for TAS with centralized configuration (CNC) management.

procedure provide a prescribed bandwidth share of the egress port using time division multiplexing. With our empirically chosen parameters, the maximum delays is capped to approximately $100 \mu$ s which is suitable for the considered topology and time-critical ST traffic that requires less than $1 \mathrm{~ms}$ of delay.

While QoS metrics are important, another factor that determines the performance gains is the admission ratio for the system. Fig. 8 shows the stream admission ratio for both reconfiguration and no reconfiguration. In general, each generated stream needs a data rate of about $11.5 \mathrm{Mbps}$ for a $50 \mu \mathrm{s}$ CT (which corresponds to approximately $45 \mu$ s of maximum ST slot size since we permit ST traffic to take up at most $90 \%$ of the CT) for each egress port on the stream's path with 1 packet injected by an ST per CT and a fixed packet size of $64 \mathrm{~B}$. With an egress port channel capacity of $R=1 \mathrm{Gbps}$, approximately 86 streams can be accommodated. Compared to the "no reconfiguration" approach, the reconfiguration approach significantly improves the admission rates at the expense of higher BE traffic delays, since the ST slot borrows BE time slots to accommodate the ST streams. We also note that increasing the maximum ST allocation above $90 \%$ would increase the ST stream admission ratio, at the expense of starving the BE traffic.

CDT traffic that requests transmission guarantees from the CNC experiences some delay before being either admitted or rejected. Since the control plane is out-of band

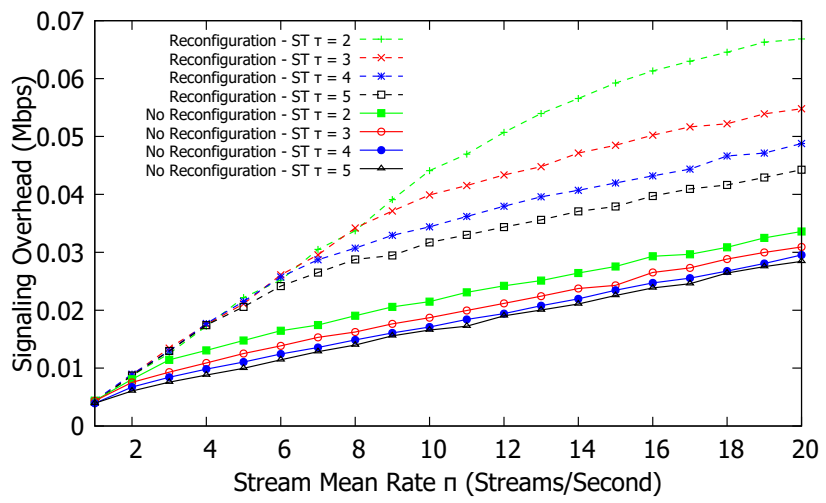

Fig. 9 - Centralized Unidirectional Topology: Stream average signaling Overhead for TAS with centralized configuration (CNC) management.

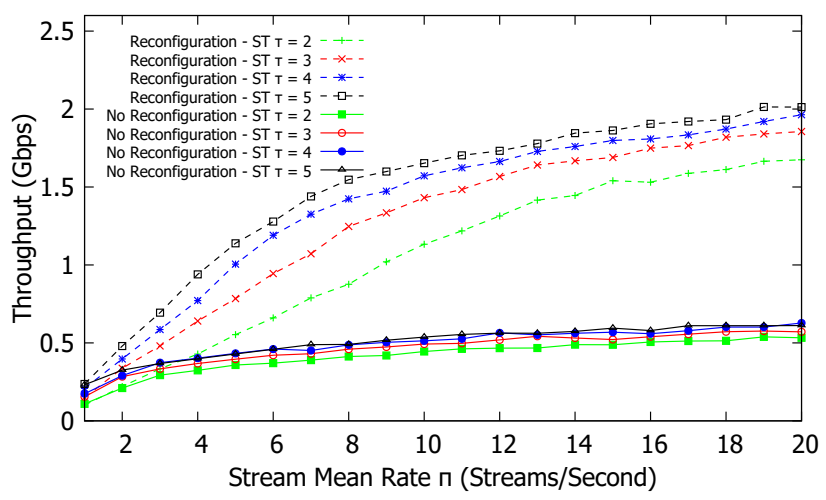

Fig. 10 - Centralized Unidirectional Topology: ST total average throughput measured at the sink for TAS with centralized configuration (CNC) management.

from the data plane within the TSN domain, the delay is constant (around $4 \mu \mathrm{s}$ ) throughout the simulation run. Stream registration and reservation introduce some control plane overhead. Fig. 9 shows the signaling overhead. More specifically, the overhead is measured as the signaling traffic rate in $\mathrm{Mbit} / \mathrm{s}$ at the $\mathrm{CNC}$ for both incoming and outgoing control (CDT) traffic. Generally, the reconfiguration introduces more signaling overhead; however, Ethernet generally has large bandwidths, thus the CDT traffic rates are minuscule compared to the link capacities. Furthermore, when $\tau=2$, we observe higher signaling overhead due to accepting larger numbers of streams (rejections are inexpensive compared to acceptance) both with and without reconfiguration.

Fig. 10 shows the average throughput measured at the sink for ST traffic. We observe from Fig. 10 that the reconfiguration substantially increases the throughput compared to the no reconfiguration scenario. Typically, the throughput is more than doubled by the reconfiguration. To examine the reliability performance, Fig. 11 shows the $\mathrm{BE}$ packet loss ratio for mid and high BE traffic loads $\rho_{L}$; we omitted the low BE traffic load which has negligible losses. Since the CNC manages only ST streams, the TSN guarantees (which include zero packet loss since retransmissions are in general too expansive for ST traffic) are only valid for ST streams. As the ST traffic load increases in the reconfiguration scenario, the BE packet loss in- 


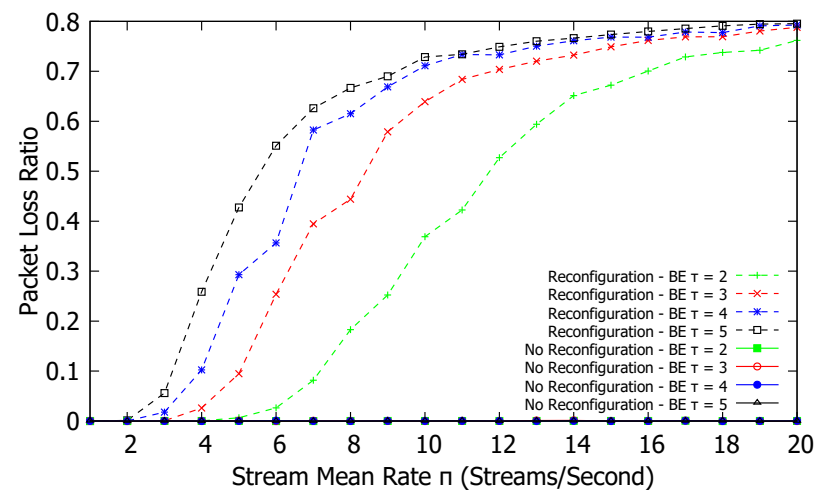

(a) Mid BE Traffic Load $\rho_{L}=1 \mathrm{Gbps}$

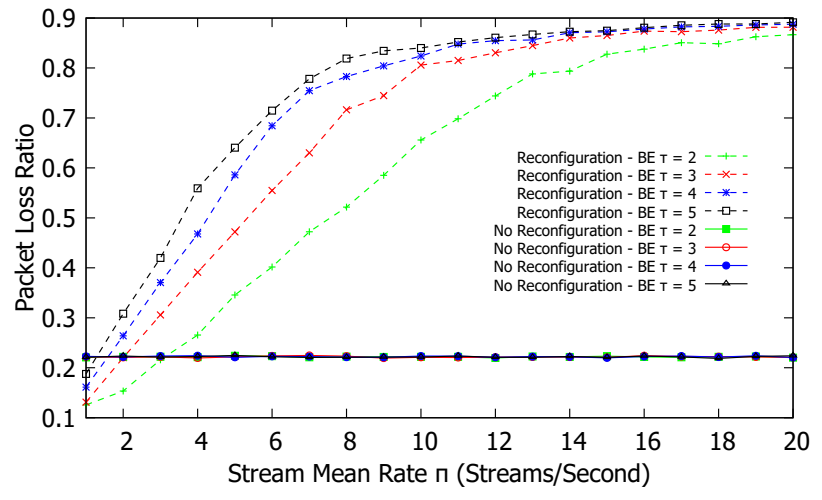

(b) High BE Traffic Load $\rho_{L}=2$ Gbps

Fig. 11 - Centralized Unidirectional Topology: BE frame loss ratio for TAS with centralized configuration (CNC) management.

creases. For the "no reconfiguration" approach, the BE packet loss is typically constant even for high loads of $\mathrm{BE}$ traffic.

For a benchmark comparison of the TSN effectiveness, and specifically TAS, we conducted additional evaluations for the scenario in Fig. 6 without the TSN slot reservation, admission control, and TAS scheduling. Specifically, we considered an ST stream mean generation rate of 1-20 streams per second with a mean lifetime $\tau=5$ seconds with the mid and high BE traffic loads of $\rho_{L}=1.0 \mathrm{Gbps}$ and $2.0 \mathrm{Gbps}$. We employed strict priority scheduling at each switch without any TSN slot reservation, i.e., each switch output port schedules and transmits all ST packets before any BE packets. We outline three main observations for the unidirectional ring topology. First, while the mean delays were generally very low for ST traffic (34$55 \mu$ s for the low traffic load range $\pi=1$ to 5 ST streams per second), the priority scheduling of the ST packets can severely starve the low-priority BE traffic (for the high $\rho_{L}=2.0 \mathrm{Gbps} \mathrm{BE}$ load, the mean BE packet delays increase from a minimum of $15 \mathrm{~ms}$ to a maximum of around $0.1 \mathrm{~s}$ as the ST load increases from 1 to 20 streams per second; whereas, with TSN, the mean BE packet delays increase from around $10 \mathrm{~ms}$ to $21 \mathrm{~ms}$, which is outside the plotted range of Fig. 6(b)). Additionally, compared to TSN, the maximum delays and jitter increase more strongly as the BE and ST loads increase (the ST maximum packet delays range from $34 \mu$ s to $20 \mathrm{~ms}$; while, with the TSN operation, the ST maximum packet delays hover around 55-101 $\mu$ s, see Fig. 7). This stronger increase of the maximum ST packet delays is a result of the BE packet traffic interfering with the ST packet traffic due to the lack of TAS operation. In particular, ST packets are blocked from transmission during an ongoing transmission of a 580 byte BE packet (as we considered non-preemptive priority scheduling). Second, since no admission control based on TSN slot reservation is used, congestion arises for ST traffic loads of $\pi=6$ to 20 ST streams per second, causing high mean and maximum delays for both ST and BE traffic. Third, due to the congestion, packet drops occur at high ST loads for both ST and BE packet traffic. We also note that since no signaling traffic is used, the priority scheduling benchmark without TSN operation provides a performance reference for both the centralized and the decentralized TSN model.

Overall, we conclude that the proposed centralized (hybrid) reconfiguration approach provides a means to ensure that dynamically varying numbers of ST streams are accommodated as permitted by the available link capacity in the unidirectional ring network. However, the unidirectional ring network does not involve any distinct routing choices towards the destination. In order to examine the performance of the proposed centralized reconfiguration in a network with different routing paths, we next consider the operation of the ring network topology as a bidirectional ring network.

\subsubsection{Bidirectional ring topology}

The unidirectional ring topology certainly simplifies the calculation of the ST slot window in the reconfiguration. In order to examine whether the proposed centralized (hybrid) reconfiguration approach can efficiently utilize the higher capacity of a more complex network with multiple routing options, we examine the bidirectional ring network. In the bidirectional ring network, each two-port switch has now two paths to the destination. We employ shortest path routing according to the hop count. We set the edge link (source to first ring switch and last ring switch to sink) capacities to $2 \mathrm{Gbps}$ to avoid congestion on the edge links (which the CNC does not control).

Fig. 12 shows the average mean ST and BE packet delay for different stream lifetimes $\tau$. Compared to the unidirectional topology (see Fig. 6), the bidirectional significantly reduces the packet delay since an extra port with full-duplex link support now provides extra capacity to service streams giving more slot reservations to BE even at high ST stream loads.

Fig. 13 shows the maximum ST packet delays for the bidirectional ring topology with CNC. We observe from Fig. 13 in comparison with the corresponding maximum packet delay plot for the unidirectional ring in Fig. 7, that the bidirectional topology with configuration gives higher maximum packet delays, which is mainly due to the substantially increasing ST stream acceptance, as examined next in Fig 14. The "no reconfiguration" keeps the ST slot size 


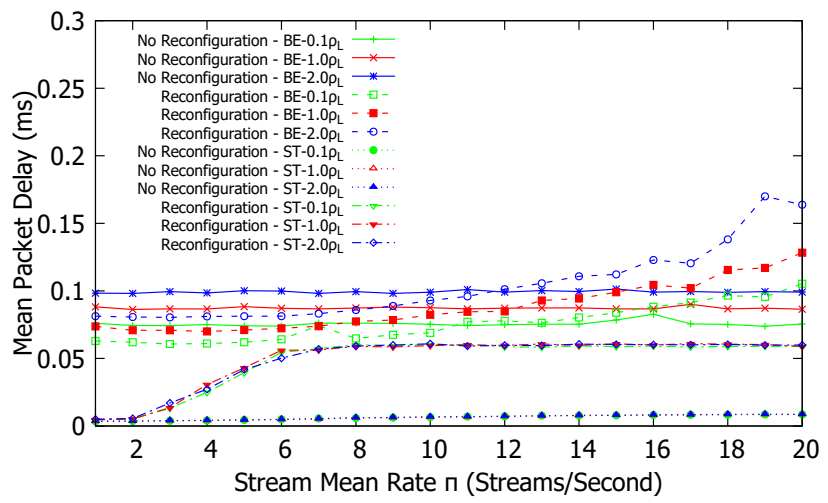

(a) $\tau=2$

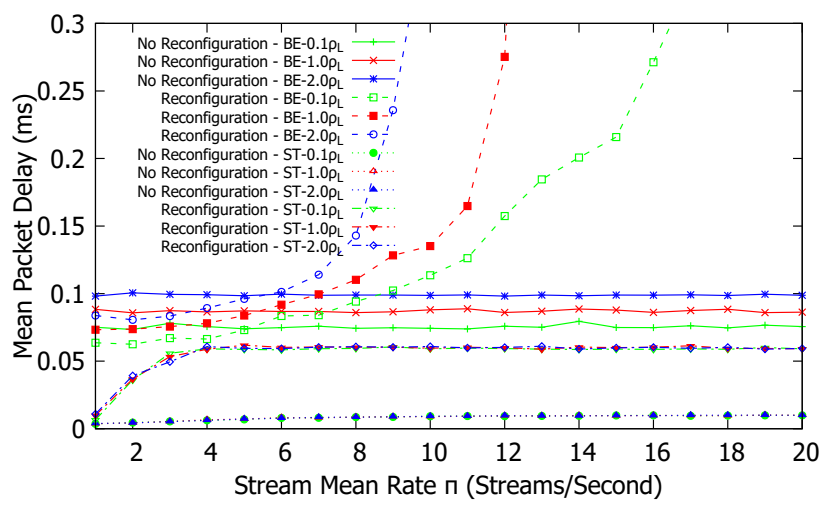

(b) $\tau=5$

Fig. 12 - Centralized Bidirectional Topology: Mean end-to-end delay for ST and BE traffic for varied mean stream lifetime $\tau$ for different BE loads $\rho_{L}$, and ST stream rates $\pi$.



Fig. 13 - Centralized Bidirectional Topology: Maximum ST packet delay for TAS with centralized configuration (CNC) management.

at the initialized value ( $20 \%$ of CT, i.e., $10 \mu \mathrm{s}$ ), resulting in a constant maximum delay of around $50 \mu \mathrm{s}$, albeit at the expense of rather low admission rates, see Fig 14.

Fig. 14 shows the stream admission ratio (percentage). With the high stream generation rate $\pi=20$ streams $/ \mathrm{s}$ and long average stream lifetime $\tau=5 \mathrm{~s}$, the admission rate is still slightly above $90 \%$ for the bidirectional topology with CNC reconfiguration. The bidirectional ring thus achieves a substantially increased (close to $50 \%$ higher) admission rate compared to the unidirectional ring examined in Fig. 8. In contrast, the increases of the admission ratio of the no reconfiguration approach with the bidirec-

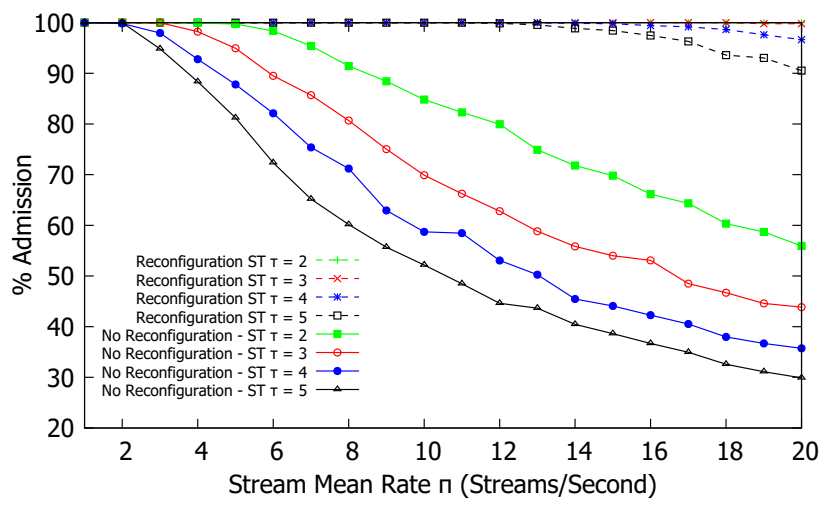

Fig. 14 - Centralized Bidirectional Topology: Stream admission percentage for TAS with centralized configuration (CNC) management.

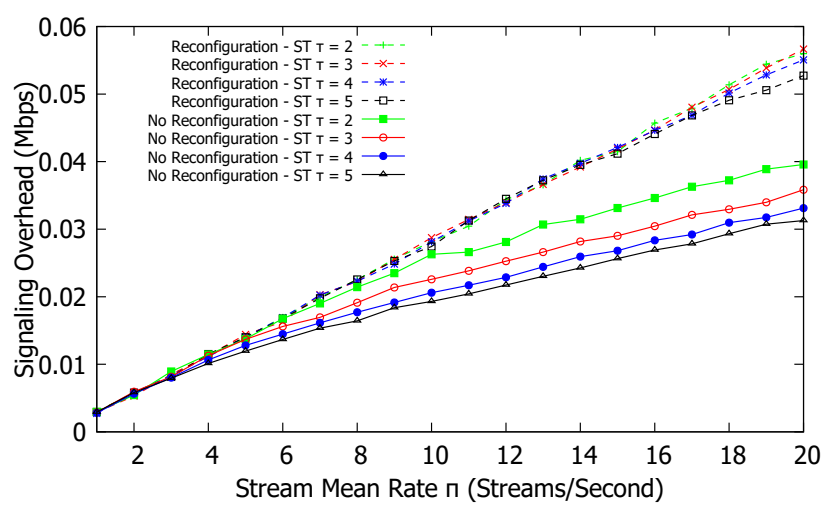

Fig. 15 - Centralized Bidirectional Topology: Average stream signaling overhead for TAS with centralized configuration (CNC) management.

tional ring compared to the unidirectional ring are more modest (roughly 20\%). This is mainly because the initialized gating ratio is too restrictive and severely underutilizes the links. We found in additional evaluations that are not included due to space constraints that different BE loads $\rho_{L}$ do not impact the ST stream performance due to the TAS operation, i.e., TAS effectively partitions the traffic at the egress switch/port (BE traffic does not block ST traffic).

Similar to the unidirectional ring, the bidirectional ring topology provides constant signaling delay (around $3.5 \mu \mathrm{s}$ ) due to the CNC out-of band signaling channels. The average signaling delay is slightly lower than in the unidirectional ring (which had a signaling delay around $4 \mu \mathrm{s}$ ), mainly since the signaling hop distances in the bidirectional ring are shorter than in the unidirectional ring. Fig. 15 shows the signaling overhead. Since the bidirectional ring topology is effectively the same as the unidirectional ring topology (albeit having another port to the switch), the signaling overhead in the bidirectional ring network is in general very similar to the signaling overhead in the unidirectional topology. Note that while the hop traversal is reduced (since the stream can take one of two paths to the destination governed by shortest path, i.e., the smallest hop count), the number of sent and received CDT frames are generally the same. Similar to the unidirectional topology, the reconfiguration approach generates more CDT traffic. Note that admissions 


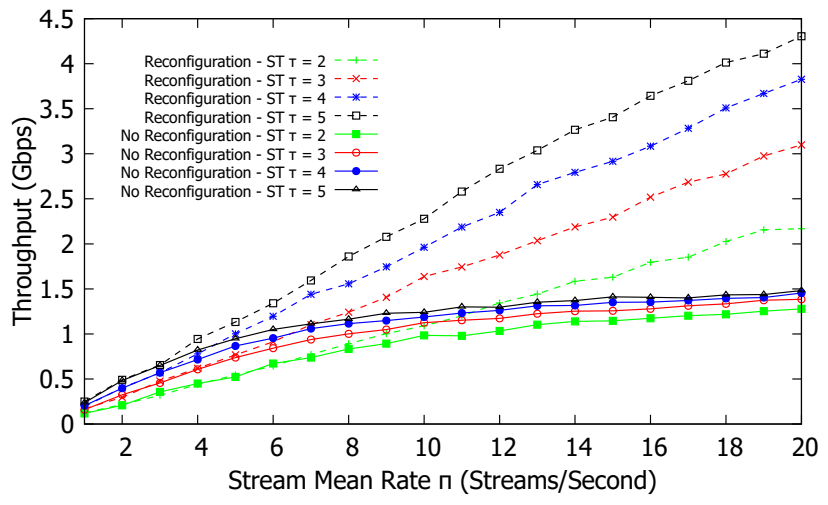

Fig. 16 - Centralized Bidirectional Topology: ST Total average throughput measured at the sink as a results of TAS with centralized configuration (CNC) management.

are in general more costly in terms of sent and received CDT frames in the network. Therefore, the higher the admission rate, the more overhead is observed in the control plane, though based on Fig. 15, the overall overhead is well below $1 \mathrm{Mbps}$ and therefore is minuscule compared to the channel capacity. We also observe from Fig. 15 that the results for different stream lifetimes $\tau$ differ only very slightly since for any $\tau$ value, almost all the streams are accepted, generating the same total overhead.

Fig. 16 shows the average overall throughput measured at the ST sinks for the bidirectional ring topology. Compared to the unidirectional ring (see Fig. 10), the throughput for the bidirectional ring is much higher, typically increased by a factor of two.

Similar to the unidirectional ring topology, the bidirectional topology achieves zero loss for ST streams while significantly reducing the BE packet loss rate. Fig. 17 shows the BE packet loss ratio for the bidirectional ring network. The maximum BE loss for the high BE traffic intensity $\rho_{L}=2.0$ is around $30 \%$ which is a significant reduction from the unidirectional topology (of around $90 \%$, see Fig. 11).

In contrast to the unidirectional topology, the bidirectional topology with central (hybrid) CNC reconfiguration achieves improved QoS metrics and admission rates. Overall, the ST traffic throughput is typically doubled in the bidirectional ring network compared to the unidirectional ring network. We can thus conclude that our proposed centralized (hybrid) CNC reconfiguration can effectively utilize the higher capacity provided by the bidirectional ring network for dynamic ST traffic, with random ST flow generations and random ST flow lifetimes.

\subsection{Decentralized model evaluation}

Analogous to the centralized (hybrid) reconfiguration evaluation, we evaluate our proposed decentralized reconfiguration from Section 4 with both periodic ST traffic and sporadic (random) BE traffic, as specified in Section 5.1.2. As before, we evaluate the network with TAS shaper on the industrial control loop unidirectional and bidirectional topology and collect results for the simula-

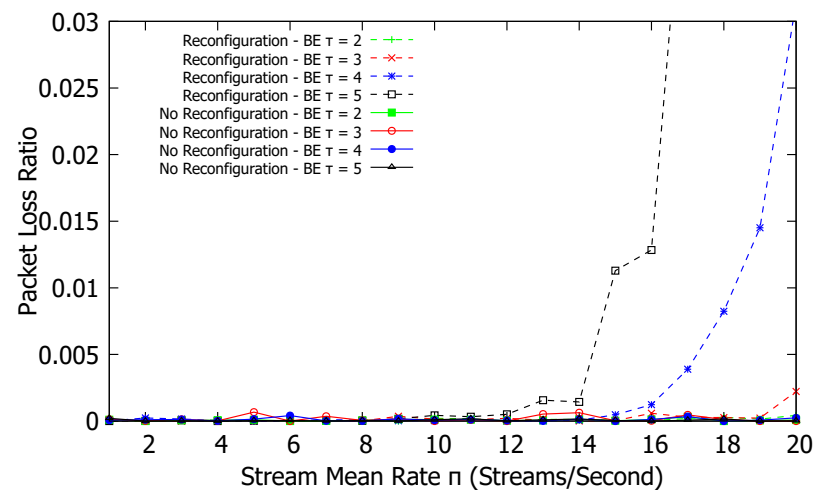

(a) Mid BE Traffic Load $\rho_{L}=1 \mathrm{Gbps}$

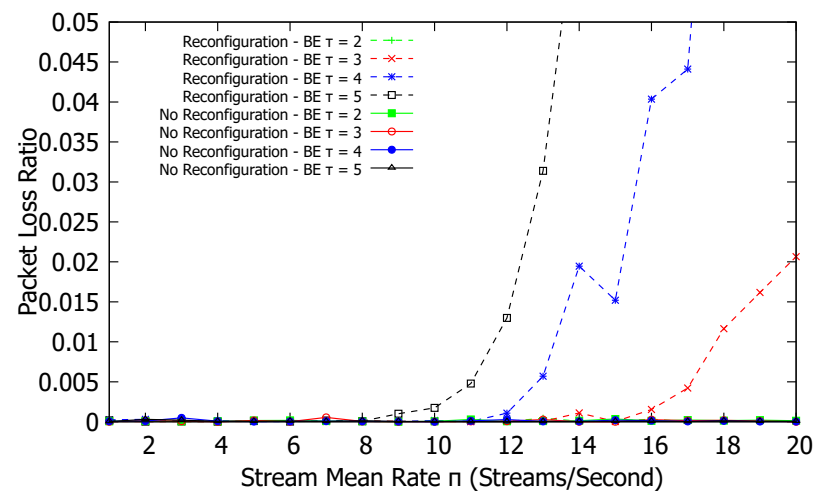

(b) High BE Traffic Load $\rho_{L}=2$ Gbps

Fig. 17 - Centralized Bidirectional Topology: BE frame loss ratio for TAS with centralized configuration (CNC) management.

tion parameters shown in Table.1.

\subsubsection{Unidirectional ring topology}

The decentralized model essentially transfers some of the CNC functions (e.g., TAS reconfiguration and resource reservation modules) from the centralized model down to the TAS enabled egress ports of the TSN switches in the data plane. The main difference between the centralized and decentralized models is the signaling performance which is now in-band and can affect data traffic. In additional evaluations that are not included due to space constraints, we have found that with the in-band CDT traffic in the decentralized model, the average ST and BE packet delays are about the same as the centralized model in Fig. 6. Typically, the ST stream's average delay is minimal to near constant for both the reconfiguration and "no reconfiguration" approaches. For BE, the "no reconfiguration" approach produces constant average delay for each BE $\rho_{L}$ traffic intensity.

Fig. 18 shows the maximum ST packet delay for the unidirectional ring network using the decentralized model. In contrast to the average ST packet delay, the maximum delay is affected by the in-band CDT traffic. In the decentralized model, the CDT traffic is given the highest priority above both ST and BE traffic. Therefore, the maximum delays can reach about $150 \mu \mathrm{s}$, which is somewhat higher than for the centralized reconfiguration in Fig. 7, but still well below $1 \mathrm{~ms}$. 


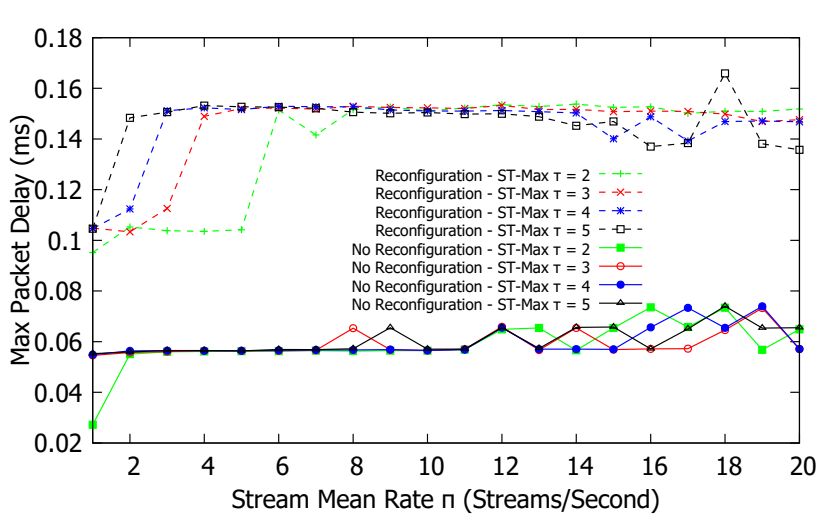

Fig. 18 - Decentralized Unidirectional Topology: Max delay for TAS.

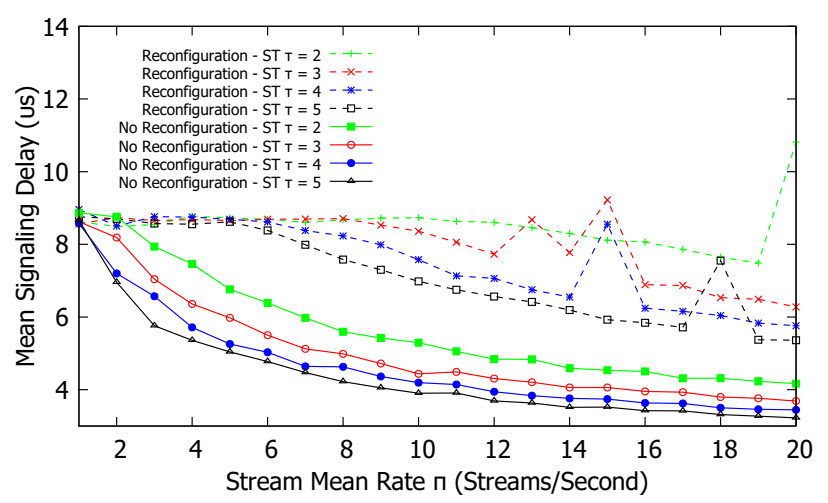

Fig. 19 - Decentralized Unidirectional Topology: Average stream signaling delay for TAS.

The stream admission rate for the decentralized model is very similar to the centralized model (see Fig. 8) and is not displayed in detail due to space constraints. Fig. 19 shows the signaling delay for ST stream registration in the decentralized model. In contrast to the centralized model, the decentralized model's in-band CDT traffic implies varied stream signaling delays. As the streams generation rate $\pi$ increases, the overall average signaling delay decreases which is due to the increased rejections as more streams attempt to request network resources. In the decentralized model, a rejection by an intermediate bottlenecked switch implies a termination of the reservation attempt and a notification to any previous pending stream records to cancel the potential reservation and eventually notify the source of the rejection. If this rejection happens closer to the source, then the average signaling delay will be shorter compared to a stream acceptance. In general, the average stream signaling delay is on the order of microseconds which is reasonable for most industrial control systems applications.

Generally, the decentralized model produced greater signaling overhead than the centralized model (cf. Fig. 9) since CDT traffic is measured at each data switch traffic port for incoming and outgoing as shown in Fig. 20. Analogous to the signaling delay, the more ST streams are accepted, the more overhead is observed. Therefore, as the stream lifetime $\tau$ increases and consequently, the more rejections occur, the lower the overhead. Overall, the comparison of the signaling overhead for the decentral-

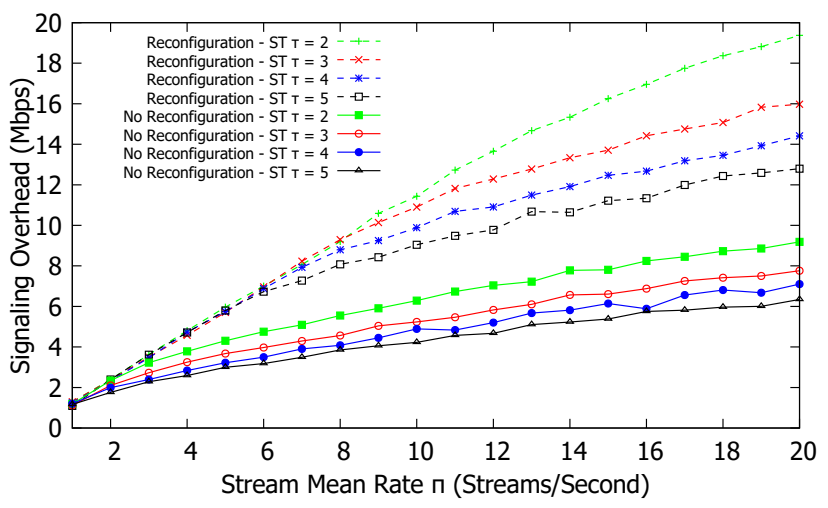

Fig. 20 - Decentralized Unidirectional Topology: Stream Signaling Overhead for TAS.

ized model in Fig. 20 with the centralized model in Fig. 9 indicates that the decentralization increases the signaling overhead by over two orders of magnitude. However, the aggregate signaling overhead bitrate in the decentralized model is still below $20 \mathrm{Mbps}$ and thus below 2\% of the 1 Gbps link capacity.

Throughput results are generally the same when compared to the unidirectional centralized model (cf. Fig. 10) and are therefore omitted. Similarly, the packet loss rate is nearly similar to the unidirectional centralized model (cf. Fig. 11). However, the unidirectional topology with either the centralized or decentralized approach generally gets bottlenecked at lower traffic loads compared to the bidirectional ring network. Therefore, BE traffic suffers as more ST streams request TAS slot reservations. We next examine the bidirectional ring network for decentralized operation to determine how the BE traffic performance can be improved while maintaining the ST traffic performance.

\subsubsection{Bidirectional ring topology}

For the bidirectional topology using the decentralized model we found that the in-band CDT traffic affects the data traffic similar to the decentralized unidirectional model, i.e., maximum ST packet delay is somewhat increased while the mean ST packet delay is essentially unchanged. As the ST stream lifetime $\tau$ is increased, i.e., the number of ST streams at any time increases, the BE slots are reallocated to ST streams which increases the mean BE packet delay which is similar to the centralized model (cf. Fig. 12) and is therefore omitted.

Fig. 21 shows the maximum ST packet delay. While the reconfiguration approach looks very similar to the centralized model (cf. Fig. 13), the no reconfiguration approach is affected by the in-band CDT traffic which raises the maximum ST packet delay in some no reconfiguration scenarios to around $100 \mu \mathrm{s}$.

The admission rate is exactly the same as for the centralized model (cf. Fig. 14). Fig. 22 shows the average signaling delay for ST stream registration. Similar to the unidirectional topology, the mean signaling delay starts to decrease as the load increases due to higher rejections. 


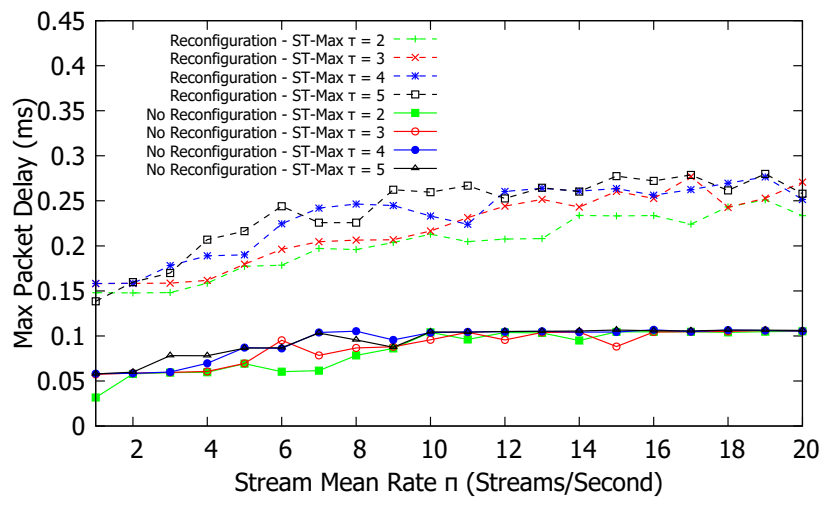

Fig. 21 - Decentralized Bidirectional Topology: Maximum ST packet delay for TAS.

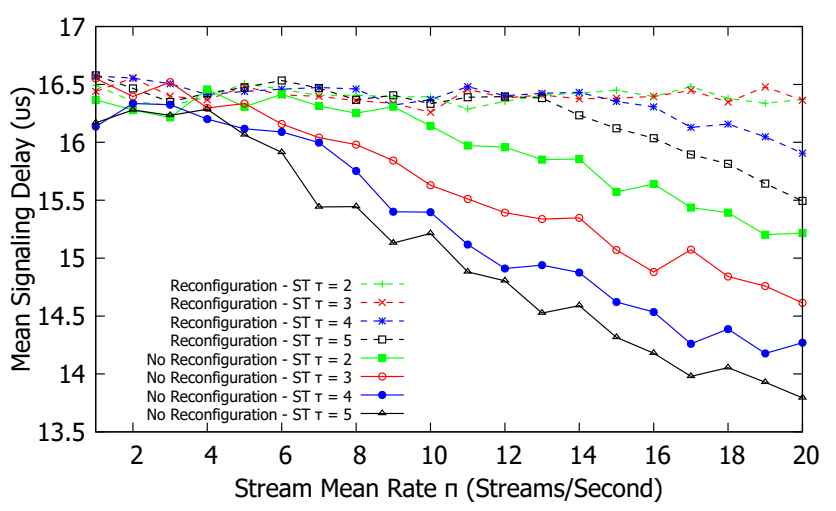

Fig. 22 - Decentralized Bidirectional Topology: Average stream signaling delay for TAS.

We found that the stream signaling overhead with the decentralized bidirectional model is similar to the decentralized unidirectional model (cf. Fig. 20), albeit slightly lower due to the shorter signaling hop counts in the bidirectional ring network.

While omitted for space, additional evaluations have found that the throughput of the bidirectional decentralized model is nearly identical to the centralized model. We observed only very slightly reduced throughput with the decentralized model compared to the centralized model since the decentralized model carries the control traffic in-band, which very slightly reduces the link utilization for data traffic.

Similar to all the preceding models and topologies, ST streams have zero traffic drops. The BE packet loss rates for the decentralized bidirectional model are nearly identical to the centralized bidirectional model. Similarly, the overall performance is largely improved under the bidirectional topology compared to the unidirectional model due to the additional port and path.

The decentralized model was found to operate nearly identically to the centralized model in terms of QoS metrics and overall admission rate. Thus, the segregation of traffic based on the class of service can be accomplished with the proposed decentralized model without the overhead complexities of a CNC node. A main disadvantage of the decentralized model is the in-band CDT traffic which can delay ST streams, particularly affecting the maximum
ST packet delays. A potential workaround to explore in future research is to service all the ST streams first, and then service CDT frames before servicing the BE traffic, though this might lead to additional signaling delays depending on the ST load.

\section{CONCLUSIONS AND FUTURE WORK}

The IEEE 802.1Qcc framework and the 802.1Qbv traffic shaper enable the implementation of a deterministic forwarding plane that provides strict bandwidth guarantees to ST flows without any flow or congestion control mechanism at the source. Using an automated network configuration is an imperative tool set to provide a unified communication platform based on commercial of the shelf (COTS) full-duplex Ethernet with high bandwidth and low complexity compared to Controller Area Networks (CANs), Local Interconnect Networks (LINs), and specialized field-buses in industrial control system applications (e.g., industrial control, automotive, and avionics). Network designs based on the IEEE 802.1Qcc framework and the $802.1 \mathrm{Qbv}$ traffic shaper can form a contract with the source to forward mission critical traffic and to automate the network configuration process using 802.1Qcc for the full lifetime of the stream. Additionally, depending on the forwarding plane port traffic shaper (e.g., TAS), the required schedules can be passed to the switch servers using general user/network information protocols (e.g., TLV, NETCONF/Yang, and SNMP).

In this paper, we have investigated the impact of TAS reconfigurations in response to dynamic network conditions, i.e., the addition and removal of transient ST streams (flows) with different lifetimes. We have demonstrated the effectiveness of TAS with and without the CNC, i.e., for centralized (hybrid) vs. decentralized (fully distributed) models. We have examined network QoS traffic characteristics when admitting ST flows based on an iterative heuristic approach that computes TAS schedules for current and newly requested ST streams.

Based on the insights from the present study we outline the following future research directions. First, it would be interesting to judiciously change the GCL time for switches during reconfiguration whilst satisfying QoS requirements. The studied reconfiguration techniques should also be examined in alternate approaches for providing deterministic QoS, e.g., [61,81] as well as in the context of related QoS oriented routing approaches, e.g. [17, 37].

Another interesting future research direction is to adapt the reconfiguration mechanisms that have been developed in this study to the interactions between TSN and fifth generation (5G) wireless communication systems that operate with Ultra-Reliable Low-Latency Communication (URLLC). A few recent studies have begun to explore the use of TSN in the 5G URLLC context, see e.g., [28, $45,52,71]$, indicating significant potential for improving 5G URLLC services by exploiting TSN. The TSN reconfiguration mechanisms developed in this study can poten- 
tially help in flexibly providing high-quality 5G URLLC services for varying traffic dynamics. Similarly, TSN reconfiguration may aid low-latency real-time services in future WiFi networks, which may incorporate TSN, see e.g., $[5,14,35]$.

In the wider context of QoS networking and related applications, deterministic networking should be examined in the context of emerging multiple-access edge computing (MEC) [21, 33, 58, 82], in particular MEC settings for low-latency applications $[23,90,94]$. As an alternative approach to coordinating the reconfigurations, emerging softwarized control and virtualization paradigms can be explored $[7,13,19,20,44,56,75,78]$. Regarding the reliability aspects, a potential future research direction is to explore low-latency network coding mechanisms, e.g., $[4,18,24,32,54,55,69]$, to enhance networking protocols targeting reliable low-latency communication.

\section{REFERENCES}

[1] IEEE Standard for Local and Metropolitan Area Networks-Virtual Bridged Local Area Networks Amendment 14: Stream Reservation Protocol (SRP). IEEE Std 802.1Qat-2010 (Revision of IEEE Std 802.1Q-2005), pages 1-119, Sept. 2010.

[2] IEEE Standard for Local and metropolitan area networks - Bridges and Bridged Networks - Amendment 25: Enhancements for Scheduled Traffic. IEEE Std 802.1Qbv-2015 (Amendment to IEEE Std 802.1Q- as amended by IEEE Std 802.1Qca-2015, IEEE Std 802.1Qcd-2015, and IEEE Std 802.1Q-/Cor 1-2015), pages 1-57, Mar. 2016.

[3] IEEE Draft Standard for Local and metropolitan area networks-Media Access Control (MAC) Bridges and Virtual Bridged Local Area Networks Amendment: Stream Reservation Protocol (SRP) Enhancements and Performance Improvements. IEEE P802.1Qcc/D2.0, October 2017, pages 1-207, Jan. 2017.

[4] J. Acevedo, R. Scheffel, S. Wunderlich, M. Hasler, S. Pandi, J. Cabrera, F. Fitzek, G. Fettweis, and M. Reisslein. Hardware acceleration for RLNC: A case study based on the Xtensa processor with the Tensilica instruction-set extension. Electronics, 7(9):180.1-180.22, 2018.

[5] T. Adame, M. Carrascosa, and B. Bellalta. TimeSensitive Networking in IEEE 802.11be: On the way to low-latency WiFi 7. arXiv preprint arXiv:1912.06086, 2019.

[6] V. Addanki and L. Iannone. Moving a step forward in the quest for Deterministic Networks (DetNet). In Proc. IFIP/IEEE Networking Conference (Networking), pages 458-466, 2020.
[7] R. Amin, M. Reisslein, and N. Shah. Hybrid SDN networks: A survey of existing approaches. IEEE Communications Surveys \& Tutorials, 20(4):3259-3306, 2018.

[8] A. Arestova, K.-S. J. Hielscher, and R. German. Design of a hybrid genetic algorithm for Time-Sensitive Networking. In Proc. Int. Conf. on Measurement, Modelling and Evaluation of Computing Sys., pages 99$117,2020$.

[9] V. Balasubramanian, M. Aloqaily, and M. Reisslein. An SDN architecture for time sensitive industrial IoT. Computer Networks, 186(107739):1-12, Feb. 2021.

[10] Belliardi, Rudy et al. Use Cases IEC/IEEE 60802, V1.3, Sept. 2018. Available from http://www.ieee802.org/1/files/public/docs2018/ 60802-industrial-use-cases-0918-v13.pdf; Last accessed Feb. 19, 2019.

[11] L. L. Bello and W. Steiner. A perspective on IEEE time-sensitive networking for industrial communication and automation systems. Proc. IEEE, 107(6):1094-1120, 2019.

[12] A. Bierman, M. Bjorklund, and K. Watsen. RESTCONF Protocol. RFC 8040, Jan. 2017.

[13] M. Böhm, J. Ohms, M. Kumar, O. Gebauer, and D. Wermser. Dynamic real-time stream reservation for IEEE 802.1 time-sensitive networks with OpenFlow. In Proc. Int. Conf. on Applied Innovations in IT, (ICAIIT), pages 7-12, 2020.

[14] D. Cavalcanti, S. Bush, M. Illouz, G. Kronauer, A. Regev, and G. Venkatesan. Wireless TSNdefinitions use cases \& standards roadmap. Avnu Alliance White Paper, pages 1-16, Mar. 2020.

[15] F. Chen. Resource Allocation Protocol (RAP) based on LRP for Distributed Configuration of Time-Sensitive Streams, 2017. http://ieee802.org/1/files/public/docs2017/tsnchen-RAP-whitepaper-0917-v01.pdf.

[16] C.-C. Chuang, T.-H. Yu, C.-W. Lin, A.-C. Pang, and T.-J. Hsieh. Online stream-aware routing for TSN-based industrial control systems. In Proc. IEEE Int. Conf. on Emerging Techn. and Factory Automation (ETFA), volume 1, pages 254-261, 2020.

[17] U. Chunduri, A. Clemm, and R. Li. Preferred Path Routing - a next-generation routing framework beyond Segment Routing. In Proc. IEEE Global Commun. Conf. (GLOBECOM), pages 1-7, Dec 2018.

[18] A. Cohen, D. Malak, V. B. Bracha, and M. Medard. Adaptive causal network coding with feedback for delay and throughput guarantees. arXiv preprint arXiv:1905.02870, 2019. 
[19] N. Deric, A. Varasteh, A. Basta, A. Blenk, R. Pries, M. Jarschel, and W. Kellerer. Coupling VNF orchestration and SDN virtual network reconfiguration. In Proc. Int. Conf. on Networked Systems (NetSys), 2019.

[20] A. Destounis, S. Paris, L. Maggi, G. S. Paschos, and J. Leguay. Minimum cost SDN routing with reconfiguration frequency constraints. IEEE/ACM Transactions on Networking, 26(4):1577-1590, 2018.

[21] T. Doan-Van, A. Kropp, G. T. Nguyen, H. Salah, and F. H. Fitzek. Programmable first: Automated orchestration between MEC and NFV platforms. In Proc. IEEE Consumer Commun. \& Netw. Conf. (CCNC), pages 1-2, 2019.

[22] T. Docquier, Y.-Q. Song, V. Chevrier, L. Pontnau, and A. Ahmed-Nacer. IEC 61850 over TSN: Traffic mapping and delay analysis of GOOSE traffic. In Proc. IEEE Int. Conf. on Emerging Techn. and Factory Automation (ETFA), volume 1, pages 246-253, 2020.

[23] M. S. Elbamby, C. Perfecto, M. Bennis, and K. Doppler. Toward low-latency and ultra-reliable virtual reality. IEEE Network, 32(2):78-84, 2018.

[24] A. Engelmann, W. Bziuk, A. Jukan, and M. Médard. Exploiting parallelism with random linear network coding in high-speed Ethernet systems. IEEE/ACM Transactions on Networking (TON), 26(6):28292842, 2018.

[25] R. Enns, M. Bjorklund, A. Bierman, and J. Schönwälder. Network Configuration Protocol (NETCONF). RFC 6241, June 2011.

[26] J. Falk, F. Dürr, and K. Rothermel. Time-triggered traffic planning for data networks with conflict graphs. In Proc. IEEE Real-Time and Embedded Techn. and Applications Symp. (RTAS), pages 124136. IEEE, 2020.

[27] J. Falk, D. Hellmanns, B. Carabelli, N. Nayak, F. Dürr, S. Kehrer, and K. Rothermel. NeSTiNg: Simulating IEEE time-sensitive networking (TSN) in OMNeT++. In Proc. IEEE Int. Conf. on Networked Systems (NetSys), pages 1-8, 2019.

[28] J. Farkas, B. Varga, G. Miklos, and J. Sachs. 5G-TSN integration meets networking requirements. Ericsson Technology Review, pages 1-11, Aug. 2019.

[29] N. Finn. IEEE Draft Standard for Local and metropolitan area networks-Media Access Control (MAC) Bridges and Virtual Bridged Local Area Networks Amendment: Link-local Registration Protocol. IEEE P802.1CS/D1.2 December 2017, Dec. 2017.

[30] N. Finn. Introduction to time-sensitive networking. IEEE Communications Standards Magazine, 2(2):2228, 2018.
[31] F. Fitzek, S.-C. Li, S. Speidel, T. Strufe, M. Simsek, and M. Reisslein. Tactile Internet with Human-in-theLoop. Academic Press, Cambridge, MA, 2021.

[32] F. Gabriel, S. Wunderlich, S. Pandi, F. H. Fitzek, and M. Reisslein. Caterpillar RLNC with feedback (CRLNC-FB): Reducing delay in selective repeat ARQ through coding. IEEE Access, 6:44787-44802, 2018.

[33] Y. Gao, W. Tang, M. Wu, P. Yang, and L. Dan. Dynamic social-aware computation offloading for lowlatency communications in IoT. IEEE Internet of Things Journal, 6(5):7864-7877, Oct. 2019.

[34] V. Gavriluţ and P. Pop. Traffic-type assignment for TSN-based mixed-criticality cyber-physical systems. ACM Transactions on Cyber-physical Systems, 4(2):1-27, 2020.

[35] E. Genc and L. F. Del Carpio. Wi-Fi QoS enhancements for downlink operations in industrial automation using TSN. In Proc. IEEE Int. Workshop on Factory Commun. Sys. (WFCS), pages 1-6, 2019.

[36] J. W. Guck, M. Reisslein, and W. Kellerer. Function split between delay-constrained routing and resource allocation for centrally managed QoS in industrial networks. IEEE Transactions on Industrial Informatics, 12(6):2050-2061, 2016.

[37] J. W. Guck, A. Van Bemten, M. Reisslein, and W. Kellerer. Unicast QoS routing algorithms for SDN: A comprehensive survey and performance evaluation. IEEE Commun. Sur. \& Tut., 20(1):388-415, First Qu. 2018.

[38] T. Häckel, P. Meyer, F. Korf, and T. C. Schmidt. Software-defined networks supporting timesensitive in-vehicular communication. arXiv preprint arXiv:1903.08039, 2019.

[39] D. Hellmanns, J. Falk, A. Glavackij, R. Hummen, S. Kehrer, and F. Dürr. On the performance of stream-based, class-based time-aware shaping and frame preemption in TSN. In 2020 IEEE International Conference on Industrial Technology (ICIT), pages 298-303. IEEE, 2020.

[40] D. Hellmanns, A. Glavackij, J. Falk, R. Hummen, S. Kehrer, and F. Dürr. Scaling TSN scheduling for factory automation networks. In Proc. IEEE Int. Conf. on Factory Commun. Systems (WFCS), pages 1$8,2020$.

[41] M. Herlich, J. L. Du, F. Schörghofer, and P. Dorfinger. Proof-of-concept for a software-defined realtime Ethernet. In Proc. IEEE Int. Conf on Emerg. Techn. and Factory Autom. (ETFA), pages 1-4, 2016.

[42] T. Hoeschele, C. Dietzel, D. Kopp, F. Fitzek, and M. Reisslein. Importance of Internet Exchange 
Point (IXP) infrastructure for 5G: Estimating the impact of $5 \mathrm{G}$ use cases. Telecommunications Policy, 45(3):102091.1-102091.18, April 2021.

[43] X. Jin, C. Xia, N. Guan, C. Xu, D. Li, Y. Yin, and P. Zeng. Real-time scheduling of massive data in time sensitive networks with a limited number of schedule entries. IEEE Access, 8:6751-6767, 2020.

[44] W. Kellerer, P. Kalmbach, A. Blenk, A. Basta, M. Reisslein, and S. Schmid. Adaptable and datadriven softwarized networks: Review, opportunities, and challenges. Proceedings of the IEEE, 107(4):711-731, April 2019.

[45] M. Khoshnevisan, V. Joseph, P. Gupta, F. Meshkati, R. Prakash, and P. Tinnakornsrisuphap. 5G industrial networks with CoMP for URLLC and time sensitive network architecture. IEEE Journal on Selected Areas in Communications, 37(4):947-959, 2019.

[46] T. Kobzan, S. Schriegel, S. Althoff, A. Boschmann, J. Otto, and J. Jasperneite. Secure and timesensitive communication for remote process control and monitoring. In Proc. IEEE Int. Conf. on Emerging Techn. and Factory Autom. (ETFA), volume 1, pages 1105-1108, 2018.

[47] M. Kovacevic, V. Skobic, M. Knezic, and Z. Ivanovic. Towards implementation of frame preemption mechanism on FPGA platform. In Proc. IEEE Int. Symp. INFOTEH-JAHORINA (INFOTEH), pages 1-7, 2020.

[48] J. Krolikowski, S. Martin, P. Medagliani, J. Leguay, S. Chen, X. Chang, and X. Geng. Joint routing and scheduling for large-scale deterministic IP networks. arXiv preprint arXiv:2004.02717, 2020.

[49] G. N. Kumar, K. Katsalis, and P. Papadimitriou. Coupling source routing with time-sensitive networking. In Proc. IFIP/IEEE Networking Conference (Networking), pages 797-802, 2020.

[50] M. Kumar, M. Boehm, J. Ohms, O. Shulha, and O. Gebauer. Evaluation of the time-aware priority queueing discipline with regard to Time-Sensitive Networking in particular IEEE 802.1Qbv. In Proc. Int. Conf. on Appl. Innovation in IT, volume 7, pages $1-6,2019$.

[51] M. Lander, P. Raagaard, M. G. Pop, and W. Steiner. Runtime reconfiguration of time-sensitive networking (TSN) schedules for fog computing. In Proc. IEEE Fog World Congress (FWC), pages 1-6, Oct. 2017.

[52] A. Larrañaga, M. C. Lucas-Estañ, I. Martinez, I. Val, and J. Gozalvez. Analysis of 5G-TSN integration to support Industry 4.0. In Proc. IEEE Int. Conf. on Emerging Techn. and Factory Automation (ETFA), volume 1, pages 1111-1114, 2020.
[53] Z. Li, H. Wan, Y. Deng, K. Xiong, and X. Song. A resource-efficient priority scheduler for timesensitive networking switches. CCF Transactions on Networking, 3:21-34, 2020.

[54] D. E. Lucani, M. V. Pedersen, D. Ruano, C. W. Sørensen, F. H. Fitzek, J. Heide, O. Geil, V. Nguyen, and M. Reisslein. Fulcrum: Flexible network coding for heterogeneous devices. IEEE Access, 6:7789077910, 2018.

[55] Z. Ma, M. Xiao, Y. Xiao, Z. Pang, H. V. Poor, and B. Vucetic. High-reliability and low-latency wireless communication for internet of things: Challenges, fundamentals and enabling technologies. IEEE Internet of Things Journal, 6(5):7946-7970, 2019.

[56] W. Mandarawi, H. Chahed, and H. de Meer. A framework for virtualizing time-aware shaper using high performance nfv. In Proc. IEEE Int. Conf. on Emerging Techn. and Factory Automation (ETFA), volume 1, pages 1621-1628, 2020.

[57] L. Martenvormfelde, A. Neumann, L. Wisniewski, and J. Jasperneite. A simulation model for integrating $5 \mathrm{G}$ into time sensitive networking as a transparent bridge. In Proc. IEEE Int. Conf. on Emerging Techn. and Factory Automation (ETFA), volume 1, pages 1103-1106, 2020.

[58] J. Martín-Pérez, L. Cominardi, C. J. Bernardos, A. De la Oliva, and A. Azcorra. Modeling mobile edge computing deployments for low latency multimedia services. IEEE Transactions on Broadcasting, 65(2):464-474, June 2019.

[59] M. A. Metaal, R. Guillaume, R. Steinmetz, and A. Rizk. Integrated industrial ethernet networks: Timesensitive networking over SDN infrastructure for mixed applications. In Proc. IFIP/IEEE Networking Conference (Networking), pages 803-808, 2020.

[60] A. Nasrallah, V. Balasubramanian, A. Thyagaturu, M. Reisslein, and H. ElBakoury. Reconfiguration algorithms for high precision communications in time sensitive networks. In Proc. IEEE Globecom Workshops (GC Wkshps), pages 1-6, 2019.

[61] A. Nasrallah, V. Balasubramanian, A. Thyagaturu, M. Reisslein, and H. ElBakoury. TSN algorithms for large scale networks: A survey and conceptual comparison. arXiv preprint arXiv:1905.08478, 2019.

[62] A. Nasrallah, A. S. Thyagaturu, Z. Alharbi, C. Wang, X. Shao, M. Reisslein, and H. ElBakoury. Performance comparison of IEEE 802.1 TSN Time Aware Shaper (TAS) and Asynchronous Traffic Shaper (ATS). IEEE Access, 7:44165-44181, 2019. 
[63] A. Nasrallah, A. S. Thyagaturu, Z. Alharbi, C. Wang, $X$. Shao, M. Reisslein, and H. ElBakoury. Ultra-low latency (ULL) networks: The IEEE TSN and IETF DetNet standards and related $5 \mathrm{G}$ ULL research. IEEE Comm. S. \& T., 21(1):88-145, 2019.

[64] J. Navarro-Ortiz, P. Romero-Diaz, S. Sendra, P. Ameigeiras, J. J. Ramos-Munoz, and J. M. LopezSoler. A survey on $5 \mathrm{G}$ usage scenarios and traffic models. IEEE Commun. Surveys \& Tutorials, 22(2):905-929, 2020.

[65] N. G. Nayak, F. Duerr, and K. Rothermel. Routing Algorithms for IEEE802. 1Qbv Networks. In Proc. RTN Workshop, ECRTS, 2017.

[66] N. G. Nayak, F. Duerr, and K. Rothermel. Routing algorithms for IEEE802.1Qbv networks. ACM SIGBED Review, 15(3):13-18, 2018.

[67] N. G. Nayak, F. Dürr, and K. Rothermel. Timesensitive software-defined network (TSSDN) for real-time applications. In Proc. ACM Int. Conf. on Real-Time Networks and Systems, pages 193-202, 2016.

[68] N. G. Nayak, F. Dürr, and K. Rothermel. Incremental Flow Scheduling \& Routing in Time-sensitive Software-defined Networks. IEEE Transactions on Industrial Informatics, 14(5):2066-2075, May 2017.

[69] V. Nguyen, E. Tasdemir, G. T. Nguyen, D. E. Lucani, F. H. P. Fitzek, and M. Reisslein. DSEP Fulcrum: Dynamic sparsity and expansion packets for Fulcrum network coding. IEEE Access, 8:78293-78314, 2020.

[70] G. S. Niemiec, L. M. S. Batista, A. E. Schaeffer-Filho, and G. L. Nazar. A survey on FPGA support for the feasible execution of virtualized network functions. IEEE Communications Surveys \& Tutorials, 22(1):504-525, 2020.

[71] J. Ohms, M. Böhm, and D. Wermser. Concept of a TSN to real-time wireless gateway in the context of $5 \mathrm{G}$ URLLC. In Proc. IEEE Int. Conf. on Wireless Networks and Mobile Commun. (WINCOM), pages 1-6, 2020.

[72] M. A. Ojewale and P. M. Yomsi. Routing heuristics for load-balanced transmission in TSN-based networks. ACM Sigbed Review, 16(4):20-25, 2020.

[73] M. A. Ojewale, P. M. Yomsi, and B. Nikolić. Multilevel preemption in TSN: Feasibility and requirements analysis. In Proc. IEEE Int. Symp. on Real-Time Distributed Computing (ISORC), pages 47-55, 2020.

[74] R. S. Oliver, S. S. Craciunas, and W. Steiner. IEEE 802.1Qbv gate control list synthesis using array theory encoding. In Proc. IEEE Real-Time and Embedded Technology and Applications Symposium (RTAS), pages 13-24, 2018.
[75] Z. Pang, X. Huang, Z. Li, S. Zhang, Y. Xu, H. Wan, and X. Zhao. Flow scheduling for conflict-free network updates in time-sensitive software-defined networks. IEEE Transactions on Industrial Informatics, 17(3):1668-1678, Mar. 2021.

[76] P. Pop, M. L. Raagaard, M. Gutierrez, and W. Steiner. Enabling fog computing for industrial automation through time-sensitive networking (TSN). IEEE Commun. Standards Mag., 2(2):55-61, 2018.

[77] W. Quan, W. Fu, J. Yan, and Z. Sun. OpenTSN: An open-source project for time-sensitive networking system development. CCF Transactions on Networking, 3(1):51-65, 2020.

[78] H. Sándor, B. Genge, and Z. Szántó. Infrastructure and framework for response and reconfiguration in Industry 4.0. In Proc. IEEE Int. Symp. on Digital Forensic and Security (ISDFS), pages 1-6, 2018.

[79] M. Schüngel, S. Dietrich, D. Ginthör, S.-P. Chen, and M. Kuhn. Analysis of time synchronization for converged wired and wireless networks. In Proc. IEEE Int. Conf. on Emerging Techn. and Factory Automation (ETFA), volume 1, pages 198-205, 2020.

[80] E. Schweissguth, D. Timmermann, H. Parzyjegla, P. Danielis, and G. Mühl. ILP-based routing and scheduling of multicast realtime traffic in timesensitive networks. In Proc. IEEE Int. Conf. on Embedded and Real-Time Computing Systems and Appl. (RTCSA), pages 1-11, 2020.

[81] M. Seaman. Paternoster policing and scheduling, Revision 2.1. May 2019. Available from http://www.ieee802.org/1/files/public/docs2019/crseaman-paternoster-policing-scheduling-0519v04.pdf, Last accessed May 25, 2019.

[82] P. Shantharama, A. S. Thyagaturu, N. Karakoc, L. Ferrari, M. Reisslein, and A. Scaglione. LayBack: SDN management of multi-access edge computing (MEC) for network access services and radio resource sharing. IEEE Access, 6:57545-57561, 2018.

[83] P. Shantharama, A. S. Thyagaturu, and M. Reisslein. Hardware-accelerated platforms and infrastructures for network functions: A survey of enabling technologies and research studies. IEEE Access, 8:132021-132085, 2020.

[84] W. Steiner, S. S. Craciunas, and R. S. Oliver. Traffic planning for time-sensitive communication. IEEE Commun. Standards Mag., 2(2):42-47, 2018.

[85] M.-T. Thi, S. B. H. Said, and M. Boc. SDN-based management solution for time synchronization in TSN networks. In Proc. IEEE Int. Conf. on Emerging Techn. and Factory Automation (ETFA), volume 1, pages 361-368, 2020. 
[86] A. Varga and R. Hornig. An overview of the OMNeT++ simulation environment. In Proc. ICST Int. Conf. on Simul. Tools and Techn. for Commun., Netw. and Sys. \& Workshops, pages 1-10, 2008.

[87] M. Vlk, Z. Hanzálek, K. Brejchová, S. Tang, S. Bhattacharjee, and S. Fu. Enhancing schedulability and throughput of time-triggered traffic in IEEE 802.1 Qbv Time-Sensitive Networks. IEEE Transactions on Communications, 68(11):7023-7038, Nov. 2020.

[88] C. von Arnim, M. Drăgan, F. Frick, A. Lechler, O. Riedel, and A. Verl. TSN-based converged industrial networks: Evolutionary steps and migration paths. In Proc. IEEE Int. Conf. on Emerging Techn. and Factory Automation (ETFA), volume 1, pages 294301, 2020.

[89] C. von Arnim, A. Lechler, O. Riedel, and A. Verl. Fragmentation in reconfigured real-time production networks. In Annals of Scientific Society for Assembly, Handling and Industrial Robotics, pages 105-115. Springer Vieweg, Berlin, Heidelberg, 2020.

[90] Z. Xiang, F. Gabriel, E. Urbano, G. T. Nguyen, M. Reisslein, and F. H. Fitzek. Reducing latency in virtual machines: Enabling tactile internet for human-machine co-working. IEEE Journal on Selected Areas in Communications, 37(5):1098-1116, 2019.

[91] J. Yan, W. Quan, X. Jiang, and Z. Sun. Injection time planning: Making CQF practical in time-sensitive networking. In Proc. IEEE INFOCOM, pages 616-625, 2020.

[92] Q. Yu and M. Gu. Adaptive group routing and scheduling in multicast time-sensitive networks. IEEE Access, 8:37855-37865, 2020.

[93] C. Zhang, Y. Wang, R. Yao, B. Zhou, L. Cheng, Y. Xu, X. Li, J. Cheng, and B. Liu. Packet-size aware scheduling algorithms in guard band for time sensitive networking. CCF Transactions on Networking, 3:4-20, 2020.

[94] K. Zhang, S. Leng, Y. He, S. Maharjan, and Y. Zhang. Mobile edge computing and networking for green and low-latency Internet of Things. IEEE Communications Magazine, 56(5):39-45, 2018.

\section{AUTHORS}

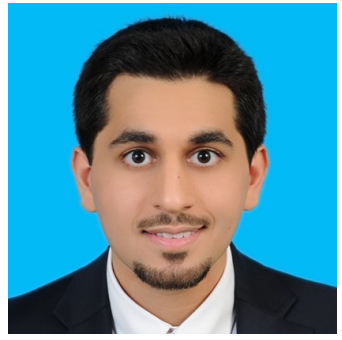

Ahmed Nasrallah is currently a researcher funded by Kuwait University to pursue the Ph.D. degree in Computer Engineering at Arizona State University, Tempe. He received his B.Sc. degree in Electrical and Computer Engineering from the University of Dayton, Dayton Ohio, and his M.S. degree in Computer Engineering from Arizona State University. His research is focused on communication and multimedia networking.

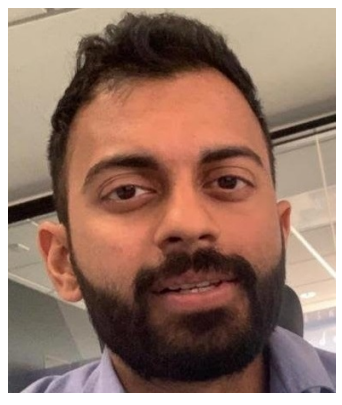

Venkatraman Balasubramanian received an MS degree in computer engineering from the University of Ottawa, in 2017. He is currently working towards his $\mathrm{PhD}$ degree in School of Electrical and Computer Engineering, Arizona State University. His research interests include edge computing, software defined network, wireless communications, and multimedia sharing over wireless networks.

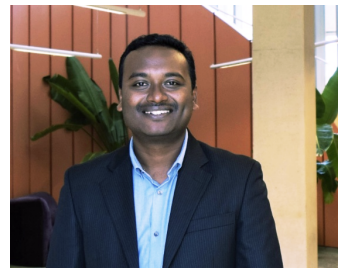

Akhilesh Thyagaturu is an Engineer at Intel Corporation, Chandler, AZ, USA, and an Adjunct Faculty in the School of Electrical, Computer, and Energy Engineering at Arizona State University (ASU), Tempe. He received a Ph.D. in electrical engineering from Arizona State University, Tempe, in 2017. He serves as a reviewer for various journals including the IEEE Communications Surveys \& Tutorials, IEEE Transactions of Network and Service Management, and Optical Fiber Technology. He was with Qualcomm Technologies Inc., San Diego, CA, USA, as an Engineer from 2013 to 2015.

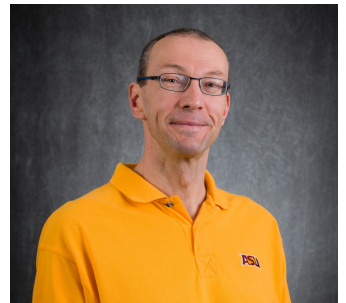

Martin Reisslein (S'96-M'98SM'03-F'14) is a Professor in the School of Electrical, Computer, and Energy Engineering at Arizona State University (ASU), Tempe. He received a Ph.D. in systems engineering from the University of Pennsylvania in 1998. He currently serves as Associate Editor for the IEEE Transactions on Mobile Computing, the IEEE Transactions on Education, and IEEE Access as well as Computer Networks and Optical Switching and Networking. He is Associate Editor-in-Chief for the IEEE Communications Surveys \& Tutorials and chairs the steering committee of the IEEE Transactions on Multimedia. 


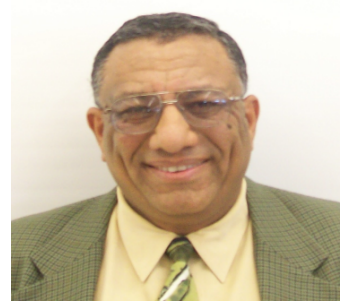

Hesham ElBakoury is a thirty five year veteran in the telecommunications and data networking industry with an extensive background and expertise in the architecture, design, and development of Distributed Systems and Broadband Access, Enterprise and Telco Communications Systems. He was a Principal Architect in Futurewei focusing on advanced technology research and standards in the network research Lab. Prior to joining Futurewei, Mr. ElBakoury was Chief Systems Architect in Hitachi-CTA EPON Access Systems Division, and Chief Systems Architect in Nortel and BellNorthern Research where he led the architecture, design, and development of several very successful Switching/Routing, Security and Carrier Ethernet products. In Nortel/BNR, Mr. ElBakoury initiated and led the Autonomic Network research project in the Enterprise division, and the Software Design and Code Reuse Project in the Data Networking division. Mr. ElBakoury has been active in different standard groups, including IEEE 802, IEEE 1904, IETF, ONF, OIF, MEF, the SCTE Energy 2020 program, and CableLabs where he has been heavily involved in IEEE 802.3/802.1, DPoE/DPoG, DOCSIS 3.1, Full-Duplex DOCSIS 3.1, SDN/NFV, Distributed CCAP Architectures, and Business Services projects. He holds an M.S.C. degree from Waterloo University, ONT, Canada. 